\title{
WEAK INTERACTION LIMITS FOR ONE-DIMENSIONAL RANDOM POLYMERS
}

March 6, 2002

\author{
Remco van der Hofstad 12 \\ Frank den Hollander 3 \\ Wolfgang König 团
}

\begin{abstract}
In this paper we present a new and flexible method to show that, in one dimension, various self-repellent random walks converge to self-repellent Brownian motion in the limit of weak interaction after appropriate space-time scaling. Our method is based on cutting the path into pieces of an appropriately scaled length, controlling the interaction between the different pieces, and applying an invariance principle to the single pieces. In this way we show that the self-repellent random walk large deviation rate function for the empirical drift of the path converges to the selfrepellent Brownian motion large deviation rate function after appropriate scaling with the interaction parameters. The method is considerably simpler than the approach followed in our earlier work, which was based on functional analytic arguments applied to variational representations and only worked in a very limited number of situations.

We consider two examples of a weak interaction limit: (1) vanishing self-repellence, (2) diverging step variance. In example (1), we recover our earlier scaling results for simple random walk with vanishing self-repellence and show how these can be extended to random walk with steps that have zero mean and a finite exponential moment. Moreover, we show that these scaling results are stable against adding self-attraction, provided the self-repellence dominates. In example (2), we prove a conjecture by Aldous for the scaling of self-avoiding walk with diverging step variance. Moreover, we consider self-avoiding walk on a two-dimensional horizontal strip such that the steps in the vertical direction are uniform over the width of the strip and find the scaling as the width tends to infinity.
\end{abstract}

2000 Mathematics Subject Classification. 60F05, 60F10, 60J55, 82D60.

Keywords and phrases. Self-repellent random walk and Brownian motion, invariance principles, large deviations, scaling limits, universality.

\footnotetext{
${ }^{1}$ Department of Applied Mathematics, Delft University of Technology, Mekelweg 4, 2628 CD Delft, The Netherlands.

${ }^{2}$ Present address: Department of Mathematics and Computer Science, Eindhoven University of Technology, P.O. Box 513, 5600 MB Eindhoven, The Netherlands. rhofstad@win.tue.nl

${ }^{3}$ EURANDOM, P.O. Box 513, 5600 MB Eindhoven, The Netherlands. denhollander@eurandom.tue.nl

${ }^{4}$ Institut für Mathematik, TU Berlin, Straße des 17. Juni 136, D-10623 Berlin, Germany. koenig@math.tu-berlin.de
} 


\section{Polymer measures}

A polymer is a long chain of atoms or molecules, often referred to as monomers, which have a tendency to repel each other. This self-repellence comes from the excluded-volume-effect: two molecules cannot occupy the same space. The self-repellence causes the polymer to spread itself out more than it would do in the absence of self-repellence. The most widely used ways to describe a polymer are the Domb-Joyce model, respectively, the Edwards model, which start from random walk, respectively, Brownian motion and build in an appropriate penalty for self-intersections. In Sections 1.1 and 1.2 we introduce these two models (in dimension one) and list some known results about their space-time scaling. In Section 2 we consider a number of variations on the Domb-Joyce model and formulate our main results, which are weak interaction limits showing that all these models scale to the Edwards model in the limit of weak interaction. Section 3 reviews some large deviation results for the Domb-Joyce model and the Edwards model, while Sections 46 contain the proofs of the theorems in Section 2. In Section 7 we close with a brief discussion of the method of proof and of some open ends.

A general background on polymers from a physics and chemistry point of view may be found in vdZ98, a survey of mathematical results for one-dimensional polymers appears in vdHK01.

\subsection{The Domb-Joyce model.}

Let $\left(S_{n}\right)_{n \in \mathbb{N}_{0}}$ be a random walk on $\mathbb{Z}$ starting at the origin $\left(S_{0}=0\right)$. Let $P$ be the law of this random walk and let $E$ be expectation with respect to $P$. Assume that the random walk is irreducible and that

$$
E\left(S_{1}\right)=0, \quad E\left(e^{\varepsilon\left|S_{1}\right|}\right)<\infty \quad \text { for some } \varepsilon>0 .
$$

Throughout the paper,

$$
\sigma^{2}=E\left|S_{1}\right|^{2} \in(0, \infty)
$$

denotes the step variance.

Fix $n \in \mathbb{N}$, introduce a parameter $\beta \in[0, \infty]$, and define a probability law $Q_{n}^{\beta}$ on $n$-step paths by setting

$$
\frac{\mathrm{d} Q_{n}^{\beta}}{\mathrm{d} P}[\cdot]=\frac{1}{Z_{n}^{\beta}} e^{-\beta H_{n}[\cdot]}, \quad Z_{n}^{\beta}=E\left(e^{-\beta H_{n}}\right),
$$

with

$$
H_{n}\left[\left(S_{i}\right)_{i=0}^{n}\right]=\sum_{\substack{i, j=0 \\ i \neq j}}^{n} \mathbb{1}_{\left\{S_{i}=S_{j}\right\}}=\sum_{x \in \mathbb{Z}} \ell_{n}(x)^{2}-(n+1)
$$

the intersection local time up to time $n$, where

$$
\ell_{n}(x)=\#\left\{0 \leq i \leq n: S_{i}=x\right\}, \quad x \in \mathbb{Z},
$$

is the local time at site $x$ up to time $n$. The law $Q_{n}^{\beta}$ is called the $n$-polymer measure with strength of self-repellence $\beta$. The path receives a penalty $e^{-2 \beta}$ for every self-intersection. The term $n+1$ in (1.4) can be trivially absorbed into the normalization.

In the case $\beta=\infty$, with the convention $e^{-\infty H_{n}}=\mathbb{1}_{\left\{H_{n}=0\right\}}$, the path measure $Q_{n}^{\infty}$ is the conditional probability law given that there are no self-intersections up to time $n$, i.e., $Q_{n}^{\infty}=P\left(\cdot \mid H_{n}=0\right)$. If single steps are equally probable under $P$, then $Q_{n}^{\infty}$ is the uniform distribution on all $n$-step selfavoiding paths having a strictly positive probability under $P$. The law $Q_{n}^{\infty}$ is known as the self-avoiding walk, and is trivial for simple random walk but non-trivial when the random walk can make larger steps. 回

\footnotetext{
${ }^{5}$ For $\beta \in(0, \infty), Q_{n}^{\beta}$ is sometimes referred to as the weakly self-avoiding walk.
} 
For the special case where

$$
S_{1} \text { is symmetric with support }\{-L, \ldots,-1,1, \ldots, L\} \text { for some } L \in \mathbb{N} \text {, }
$$

the following is known.

Theorem 1.1 (CLT and partition function). Fix $\beta \in[0, \infty]$, assume (1.6), and exclude the trivial case $(\beta, L)=(\infty, 1)$. Then there are numbers $r^{*}, \theta^{*}, \sigma^{*} \in(0, \infty)$ (depending on $\beta$ and on the distribution of $\left.S_{1}\right)$ such that:

(i) Under the law $Q_{n}^{\beta}$, the distribution of the scaled and normalized endpoint $\left(\left|S_{n}\right|-\theta^{*} n\right) / \sigma^{*} \sqrt{n}$ converges weakly to the standard normal distribution.

(ii) $\lim _{n \rightarrow \infty} \frac{1}{n} \log Z_{n}^{\beta}=-r^{*}$.

Theorem 1.1(i) is contained in [K96, Theorem 1.1], Theorem 1.1(ii) is proved in [K94] for $\beta<\infty$ and in K93 for $\beta=\infty$. For $L=1$, the law of large numbers contained in Theorem 1.1(i) first appeared in Greven and den Hollander GH93.

\subsection{The Edwards model.}

Let $B=\left(B_{t}\right)_{t>0}$ be a standard Brownian motion on $\mathbb{R}$ starting at the origin $\left(B_{0}=0\right)$. Let $\widehat{P}$ be the Wiener measure and let $\widehat{E}$ be expectation with respect to $\widehat{P}$. For $T>0$ and $\beta \in[0, \infty)$, define a probability law $\widehat{Q}_{T}^{\beta}$ on paths of length $T$ by setting

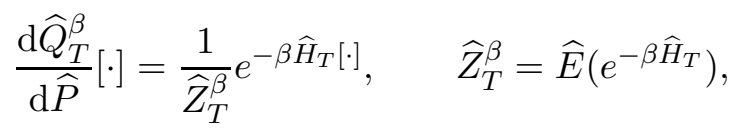

with

$$
\widehat{H}_{T}\left[\left(B_{t}\right)_{t \in[0, T]}\right]=\int_{0}^{T} \mathrm{~d} u \int_{0}^{T} \mathrm{~d} v \delta\left(B_{u}-B_{v}\right)=\int_{\mathbb{R}} L(T, x)^{2} \mathrm{~d} x
$$

the Brownian self-intersection local time up to time $T$. The middle expression in (1.8) is formal only. In the last expression the Brownian local times $L(T, x), x \in \mathbb{R}$, appear. The law $\widehat{Q}_{T}^{\beta}$ is called the $T$-polymer measure with strength of self-repellence $\beta$. The Brownian scaling property implies that

$$
(L(t, x))_{t \in[0, T], x \in \mathbb{R}} \stackrel{\mathcal{D}}{=}\left(\beta^{-\frac{1}{3}} L\left(\beta^{\frac{2}{3}} t, \beta^{\frac{1}{3}} x\right)\right)_{t \in[0, T], x \in \mathbb{R}}, \quad \beta, T>0
$$

(here $\stackrel{\mathcal{D}}{=}$ means equal in distribution under $\widehat{P}$ ), and hence that

$$
\widehat{Q}_{T}^{\beta}\left(\left(B_{t}\right)_{t \in[0, T]} \in \cdot\right)=\widehat{Q}_{\beta^{\frac{2}{3}} T}^{1}\left(\left(\beta^{-\frac{1}{3}} B_{\beta^{\frac{2}{3}} t}\right)_{t \in[0, T]} \in \cdot\right), \quad \beta, T>0 .
$$

Theorem 1.2 (CLT and partition function). There are numbers $a^{*}, b^{*}, c^{*} \in(0, \infty)$ such that, for any $\beta \in(0, \infty)$ :

(i) Under the law $\widehat{Q}_{T}^{\beta}$, the distribution of the scaled and normalized endpoint $\left(\left|B_{T}\right|-b^{*} \beta^{\frac{1}{3}} T\right) / c^{*} \sqrt{T}$ converges weakly to the standard normal distribution.

(ii) $\lim _{T \rightarrow \infty} \frac{1}{T} \log \widehat{Z}_{T}^{\beta}=-a^{*} \beta^{\frac{2}{3}}$.

Theorem 1.2 is proved in vdHdHK97a. Rigorous bounds on $a^{*}, b^{*}, c^{*}$ appeared in $\mathrm{vdH98}$, Theorem $3]$. The numerical values are: $a^{*} \approx 2.19, b^{*} \approx 1.11, c^{*} \approx 0.63$. The law of large numbers contained in Theorem 1.2(i) first appeared in Westwater W84. 


\section{MAin RESUltS}

In this section we formulate and explain our main results, all of which are weak interaction limits for the large space-time scaling of the one-dimensional Domb-Joyce model introduced in Section 1.1 and various related models. In all cases the scaling is the same as that of the Edwards model introduced in Section 1.2, showing that universality holds. Two examples of a weak interaction limit are considered: $\beta \downarrow 0$ and $\sigma \rightarrow \infty$.

Section 2.1 considers the Domb-Joyce model, Section 2.2 the Domb-Joyce model with added selfattraction, and Section 2.3 self-avoiding walk on a two-dimensional strip. In Section 2.4 we describe some invariance principles that are needed in the proofs appearing in Sections 16 . A brief discussion of our results and our method of proof can be found in Section 0 .

\subsection{Two weak interaction limits for self-repellent polymers.}

Consider an arbitrary random walk $\left(S_{n}\right)_{n \in \mathbb{N}_{0}}$ on $\mathbb{Z}$ satisfying (1.1), respectively, the two technical conditions 2.232 .24 ) introduced in Section 2.4 .

\section{Theorem 2.1 (LLN).}

(i) Fix $\sigma \in(0, \infty)$. Then, under (1.1),

$$
\lim _{\beta \downarrow 0} \limsup _{n \rightarrow \infty} Q_{n}^{\beta}\left(\left|\frac{\left|S_{n}\right|}{\beta^{\frac{1}{3}} n}-b^{*} \sigma^{\frac{2}{3}}\right| \geq \varepsilon\right)=0 \quad \forall \varepsilon>0 .
$$

(ii) Fix $\beta=\infty$. Then, under (2.23)-(2.24),

$$
\lim _{\sigma \rightarrow \infty} \limsup _{n \rightarrow \infty} Q_{n}^{\infty}\left(\left|\frac{\left|S_{n}\right|}{\sigma^{\frac{2}{3}} n}-b^{*}\right| \geq \varepsilon\right)=0 \quad \forall \varepsilon>0 .
$$

Theorem 2.1 is proved in Sections 4 5. It is to be viewed as an approximative law of large numbers for the endpoint $S_{n}$ of the polymer, since it states that the asymptotics of $\left|S_{n}\right| / n$ as $n \rightarrow \infty$ behaves like $b^{*} \sigma^{\frac{2}{3}} \beta^{\frac{1}{3}}$ as $\beta \downarrow 0$, respectively, like $b^{*} \sigma^{\frac{2}{3}}$ as $\sigma \rightarrow \infty$. Note that in Theorem 2.1](i) the asymptotics does not depend on the details of the random walk other than its step variance.

In the special case of (1.6), where the central limit theorem is known (recall Theorem 1.1(i)), we obtain the following two corollaries for the scaling of the parameters $r^{*}$ and $\theta^{*}$ as $\beta \downarrow 0$, respectively, $\sigma \rightarrow \infty$. To stress this dependence, we write $r^{*}=r^{*}(\beta), \theta^{*}=\theta^{*}(\beta)$. Both these corollaries are also proved in Sections 6 田.

Corollary 2.2 (Scaling rate and drift). Fix $\sigma \in(0, \infty)$. Then, under (1.6),

$$
r^{*}(\beta) \sim a^{*} \sigma^{-\frac{2}{3}} \beta^{\frac{2}{3}}, \quad \theta^{*}(\beta) \sim b^{*} \sigma^{\frac{2}{3}} \beta^{\frac{1}{3}}, \quad \beta \downarrow 0 .
$$

For the nearest-neighbor random walk $\left(\sigma^{2}=1\right)$, the assertions in Corollary 2.2 were already proved in vdHdH95, Theorems 4-6]. However, the proof used heavy functional analytic tools and gave no probabilistic insight. For $\sigma^{2}>1$ this route seems inaccessible, so it is nice that here the scaling comes out more generally.

Corollary 2.3 (Scaling rate and drift). Fix $\beta=\infty$. Then, under (1.6) and (2.23)-(2.24),

$$
r^{*}(\infty) \sim a^{*} \sigma^{-\frac{2}{3}}, \quad \theta^{*}(\infty) \sim b^{*} \sigma^{\frac{2}{3}}, \quad \sigma \rightarrow \infty .
$$


The second assertion in Corollary 2.3 settles a conjecture due to Aldous [A86, Section 7(B)], although Aldous misses the factor $b^{*}$.

We believe that also

$$
\sigma^{*}(\beta) \rightarrow c^{*}, \quad \beta \downarrow 0, \quad \text { respectively } \quad \sigma^{*}(\infty) \rightarrow c^{*}, \quad \sigma \rightarrow \infty,
$$

but we are unable to prove this. The reason why will become clear in Section 4.2. For nearest-neighbor random walk, the first assertion in (2.5) was proved in vdHdHK97b.

Our approach is flexible enough to allow for a coupled limit $n \rightarrow \infty$ and $\beta \downarrow 0$, respectively, $\sigma \rightarrow \infty$.

Theorem 2.4 (Coupled LLN).

(i) Fix $\sigma \in(0, \infty)$, and assume (11.1). If $\beta$ is replaced by $\beta_{n}$ satisfying $\beta_{n} \rightarrow 0$ and $\beta_{n} n^{\frac{3}{2}} \rightarrow \infty$ as $n \rightarrow \infty$, then

$$
\lim _{n \rightarrow \infty} Q_{n}^{\beta_{n}}\left(\left|\frac{\left|S_{n}\right|}{\beta_{n}^{\frac{1}{3}} n}-b^{*} \sigma^{\frac{2}{3}}\right| \geq \varepsilon\right)=0 \quad \forall \varepsilon>0 .
$$

(ii) Fix $\beta=\infty$, and assume (1.6) and (2.23)-2.24). If $\sigma$ is replaced by $\sigma_{n}$ satisfying $\sigma_{n} \rightarrow \infty$ and $\sigma_{n} n^{-\frac{3}{2}} \rightarrow 0$ as $n \rightarrow \infty$, then

$$
\lim _{n \rightarrow \infty} Q_{n}^{\infty}\left(\left|\frac{\left|S_{n}\right|}{\sigma_{n}^{\frac{2}{3}} n}-b^{*} \beta^{\frac{1}{3}}\right| \geq \varepsilon\right)=0 \quad \forall \varepsilon>0 .
$$

Theorem 2.4 is proved in Section 6.1. For simple random walk $\left(\sigma^{2}=1\right)$, the assertion in Theorem 2.4(i) was already proved in vdHdHK97b, Theorem 1.5]. Note that the conditions on $\beta_{n}$, respectively, $\sigma_{n}$ keep the scaling out of the central limit regime.

\subsection{Weak interaction limit for self-repellent and self-attractive polymers.}

The method introduced in this paper extends to the situation where self-attraction is added to the polymer. In (1.3), we replace $\beta H_{n}$ by

$$
\begin{aligned}
H_{n}^{\beta, \gamma} & =\beta \sum_{\substack{i, j=0 \\
i \neq j}}^{n} \mathbb{1}_{\left\{S_{i}=S_{j}\right\}}-\frac{\gamma}{2} \sum_{\substack{i, j=0 \\
i \neq j}}^{n} \mathbb{1}_{\left\{\left|S_{i}-S_{j}\right|=1\right\}} \\
& =(\beta-\gamma) \sum_{x \in \mathbb{Z}} \ell_{n}^{2}(x)+\frac{\gamma}{2} \sum_{x \in \mathbb{Z}}\left[\ell_{n}(x)-\ell_{n}(x+1)\right]^{2}-\beta(n+1),
\end{aligned}
$$

where $\beta, \gamma \in(0, \infty)$ are parameters, and $\left(S_{n}\right)_{n \in \mathbb{N}_{0}}$ is an arbitrary random walk on $\mathbb{Z}$ satisfying (1.1). In words, $H_{n}^{\beta, \gamma}$ is equal to $\beta$ times twice the number of self-intersections up to time $n$ minus $\gamma$ times twice the number of self-contacts up to time $n$. The law $Q_{n}^{\beta, \gamma}$ gives a penalty $e^{-2 \beta}$ to every pair of monomers at the same site and a reward $e^{\gamma}$ to every pair of monomers at neighboring sites. The term $\beta(n+1)$ in (2.8) can again be trivially absorbed into the normalization.

The scaling behavior under $Q_{n}^{\beta, \gamma}$ was studied (in arbitary dimension) in vdHK00. It was shown that there is a phase transition at $\beta=\gamma$, namely, the polymer collapses on a finite (random) number of sites when $\gamma>\beta$, while it visits order $n$ sites when $\gamma<\beta$. Furthermore, in dimension one, a law of large numbers and a central limit theorem for the endpoint $S_{n}$ under $Q_{n}^{\beta, \gamma}$, analogous to Theorem 1.1(i), were derived under the restriction $0<\gamma<\beta-\frac{1}{2} \log 2$.

We want to obtain the analogue of Theorem 2.1(i). In Theorem 2.5 below we abbreviate

$$
\lim _{\beta, \gamma} \quad \text { for } \quad \beta, \gamma \downarrow 0 \text { such that } 0<\gamma<\beta \text { and } \gamma(\beta-\gamma)^{-\frac{2}{3}} \rightarrow 0,
$$

and likewise for liminf and limsup. 
Theorem 2.5 (LLN). Fix $\sigma \in(0, \infty)$. Then, under (1.1),

$$
\lim _{\beta, \gamma} \limsup _{n \rightarrow \infty} Q_{n}^{\beta, \gamma}\left(\left|\frac{\left|S_{n}\right|}{(\beta-\gamma)^{\frac{1}{3}} n}-b^{*} \sigma^{\frac{2}{3}}\right| \geq \varepsilon\right)=0 \quad \forall \varepsilon>0 .
$$

Theorem 2.5 is proved in Section 6.2. Note that no law of large numbers is known for small $\beta, \gamma$. If

$$
\theta^{*}(\beta, \gamma)=\lim _{n \rightarrow \infty} E_{Q_{n}^{\beta, \gamma}}\left(\frac{\left|S_{n}\right|}{n}\right) \in(0, \infty)
$$

would exist for fixed $\beta, \gamma$, then we could deduce from Theorem 2.5 that $\lim _{\beta, \gamma}(\beta-\gamma)^{-\frac{1}{3}} \theta^{*}(\beta, \gamma)=b^{*} \sigma^{\frac{2}{3}}$.

We believe that Theorem 2.5 fails without the restrictions on $\beta, \gamma$ in (2.9). There is also a coupled limit version of Theorem 2.5 analogous to Theorem 2.4, but we refrain from writing this down.

\subsection{Weak interaction limit for self-avoiding polymers on a two-dimensional strip.}

Let $\left(X_{n}\right)_{n \in \mathbb{N}_{0}}=\left(S_{n}, U_{n}^{L}\right)_{n \in \mathbb{N}_{0}}$ be a random walk on the strip $\mathbb{Z} \times\{-L, \ldots, L\}$, where $\left(S_{n}\right)_{n \in \mathbb{N}_{0}}$ is a random walk on $\mathbb{Z}$ satisfying (1.1), and $\left(U_{n}^{L}\right)_{n \in \mathbb{N}_{0}}$ is an i.i.d. sequence, independent of $\left(S_{n}\right)_{n \in \mathbb{N}_{0}}$, such that $U_{0}^{L}$ is uniformly distributed on $\{-L, \ldots, L\}$. For this two-dimensional random walk, define its self-avoiding version by putting $Q_{n}^{\infty, L}(\cdot)=P^{L}\left(\cdot \mid H_{n}=0\right)$, where $P^{L}$ is the law of $\left(X_{n}\right)_{n \in \mathbb{N}_{0}}$ and

$$
H_{n}=\sum_{\substack{i, j=0 \\ i \neq j}}^{n} \mathbb{1}_{\left\{X_{i}=X_{j}\right\}}
$$

is the intersection local time up to time $n$.

Theorem 2.6 below identifies the asymptotics of the endpoint of the first coordinate, $S_{n}$, under the law $Q_{n}^{\infty, L}$ in the limit as $n \rightarrow \infty$ followed by $L \rightarrow \infty$, and also when the two limits are coupled.

Theorem 2.6 (LLN and coupled LLN). Fix $\sigma \in(0, \infty)$ and assume (1.1).

(i) Then

$$
\lim _{L \rightarrow \infty} \limsup _{n \rightarrow \infty} Q_{n}^{\infty, L}\left(\left|\frac{\left|S_{n}\right|}{(4 L)^{-\frac{1}{3}} n}-b^{*} \sigma^{\frac{2}{3}}\right| \geq \varepsilon\right)=0 \quad \forall \varepsilon>0 .
$$

(ii) If $L$ is replaced by $L_{n}$ satisfying $L_{n} \rightarrow \infty$ and $L_{n} n^{-\frac{3}{2}} \rightarrow 0$ as $n \rightarrow \infty$, then

$$
\lim _{n \rightarrow \infty} Q_{n}^{\infty, L_{n}}\left(\left|\frac{\left|S_{n}\right|}{\left(4 L_{n}\right)^{-\frac{1}{3}} n}-b^{*} \sigma^{\frac{2}{3}}\right| \geq \varepsilon\right)=0 \quad \forall \varepsilon>0 .
$$

Theorem 2.6 is proved in Section 6.3. In [AJ90, it is shown that

$$
\theta^{*}(L)=\lim _{n \rightarrow \infty} E_{Q_{n}^{\infty, L}}\left(\frac{\left|S_{n}\right|}{n}\right) \in(0, \infty)
$$

exists for fixed $L$. Therefore, we deduce from Theorem 2.6(i) that $\lim _{L \rightarrow \infty}(4 L)^{\frac{1}{3}} \theta^{*}(L)=b^{*} \sigma^{\frac{2}{3}}$.

We close this section by making a comparison with self-avoiding walk on $\mathbb{Z}^{2}$. One of the prominent open problems for this process is the asymptotic analysis of its endpoint. The conjecture is that the endpoint runs on scale $n^{\frac{3}{4}}$. Now, interestingly, in Theorem 2.6(ii) it is precisely the choice $L_{n}=n^{\frac{3}{4}}$ that makes the two coordinates $S_{n}$ and $U_{n}^{L_{n}}$ run on the same scale $n^{\frac{3}{4}}$. This suggests that for $L_{n}=n^{\frac{3}{4}}$ the behavior on the strip is a reasonable qualitative approximation to the behavior on $\mathbb{Z}^{2}$.

Let us try to make this argument a bit more precise by appealing to an adaptation of the well-known Flory argument (see MS93, Section 2.2]). Let $S=\left(S_{n}\right)_{n \in \mathbb{N}_{0}}=\left(S_{n}^{(1)}, S_{n}^{(2)}\right)_{n \in \mathbb{N}_{0}}$ be two-dimensional 
simple random walk. We may assume that $S^{(1)}=\left(S_{n}^{(1)}\right)_{n \in \mathbb{N}_{0}}$ and $S^{(2)}=\left(S_{n}^{(2)}\right)_{n \in \mathbb{N}_{0}}$ are two independent one-dimensional simple random walks. f We want to investigate the quantity

$$
\begin{aligned}
Z_{n}^{\infty}(\nu) & =P\left(\bigcap_{\substack{i, j=0 \\
i \neq j}}^{n}\left\{S_{i} \neq S_{j}\right\} \cap\left\{\left|S_{n}\right| \asymp n^{\nu}\right\}\right) \\
& =E^{(1)}\left(\mathbb{1}\left\{\left|S_{n}^{(1)}\right| \asymp n^{\nu}\right\} P\left(\bigcap_{\substack{i, j=0 \\
i \neq j}}^{n}\left\{S_{i} \neq S_{j}\right\} \cap\left\{\left|S_{n}^{(2)}\right| \asymp n^{\nu}\right\} \mid S^{(1)}\right)\right),
\end{aligned}
$$

where $P$ is the law of $S, E^{(1)}$ is expectation with respect to $S^{(1)}$, and $\nu>0$ is an exponent to be determined later. Denote the local times of $S^{(1)}$ by $\ell_{n}^{(1)}(x), x \in \mathbb{Z}$. Note that $S^{(1)}$ has $\ell_{n}^{(1)}(x)\left[\ell_{n}^{(1)}(x)-1\right]$ self-intersections at $x \in \mathbb{Z}$. In order that $S$ has no self-intersections, $S^{(2)}$ must avoid a self-intersection at the $\sum_{x \in \mathbb{Z}} \ell_{n}^{(1)}(x)\left[\ell_{n}^{(1)}(x)-1\right]$ time pairs at which $S^{(1)}$ has self-intersections. Now, let us make the crude approximation that $S_{i}^{(2)}, i=0, \ldots, n$, are i.i.d. uniformly distributed on $\left\{-\left|S_{n}^{(2)}\right|, \ldots,\left|S_{n}^{(2)}\right|\right\}$. Then, on the event $\left\{\left|S_{n}^{(2)}\right| \asymp n^{\nu}\right\}$, the probability that a self-intersection of $S^{(2)}$ occurs at a given time pair $i \neq j$ at which $S_{i}^{(1)}=S_{j}^{(1)}$ is $\asymp n^{-\nu}$. (The idea behind the approximation is that for large $n$ most self-intersections occur when $|i-j|$ is large.) The resulting model is precisely the one investigated in Theorem 2.6(ii) with $L_{n} \asymp n^{\nu}$. For this choice, (2.14) yields that $\left\{S_{n}^{(2)} \asymp n^{1-\frac{\nu}{3}}\right\}$ is typical. Putting $\nu=1-\frac{1}{3} \nu$, we find $\nu=\frac{3}{4}$.

\subsection{Invariance principles and assumptions on variance scaling.}

The proofs of our weak interaction limits in Sections 2.12 .3 will be based on a number of invariance principles, which we describe now. Let $\left(B_{t}^{\sigma}\right)_{t \geq 0}$ be a Brownian motion with generator $\frac{1}{2} \sigma^{2} \Delta$, and write $\widehat{H}_{T}^{\sigma}$ for its intersection local time and $L^{\sigma}(T, x), x \in \mathbb{R}$, for its local times up to time $T$.

I. The first invariance principle we will rely on was put forward in [BS95, Theorem 1.3]: 1]

$$
\left(n^{-\frac{1}{2}}\left(S_{\lfloor n t\rfloor}\right)_{t \in[0, T]}, n^{-\frac{3}{2}} H_{\lfloor n T\rfloor}\right) \stackrel{n \rightarrow \infty}{\Longrightarrow}\left(\left(B_{t}^{\sigma}\right)_{t \in[0, T]}, \widehat{H}_{T}^{\sigma}\right), \quad \sigma, T>0 .
$$

This says that the Domb-Joyce model (for the random walk with variance $\sigma^{2}$ ) at time $n T$ with strength of self-repellence $\beta n^{-\frac{3}{2}}$ converges, after appropriate space-time scaling, to the Edwards model (for the Brownian motion with generator $\left.\frac{1}{2} \sigma^{2} \Delta\right)$ at time $T$ with strength of self-repellence $\beta$. Another version of the same invariance principle is the assertion

$$
\left(\beta^{\frac{1}{3}}\left(S_{\left\lfloor\beta^{-\frac{2}{3}} t\right\rfloor}\right)_{t \in[0, T]}, \beta H_{\left\lfloor\beta^{-\frac{2}{3}} T\right]}\right) \stackrel{\beta \downarrow 0}{\Longrightarrow}\left(\left(B_{t}^{\sigma}\right)_{t \in[0, T]}, \widehat{H}_{T}^{\sigma}\right), \quad \sigma, T>0 .
$$

As was shown in CR83, the discrete local times process converges weakly to the continuous local times process:

$$
\left(\beta^{\frac{1}{3}} \ell_{\left\lfloor\beta^{-\frac{2}{3}} T\right\rfloor}\left(\left\lfloor x \beta^{-\frac{1}{3}}\right\rfloor\right)\right)_{x \in \mathbb{R}} \stackrel{\beta \downarrow 0}{\longrightarrow}\left(L^{\sigma}(T, x)\right)_{x \in \mathbb{R}}, \quad \sigma, T>0 .
$$

This explains the scaling of the second component in $(2.17)-(2.18)$. Since $\left(B_{t}^{\sigma}\right)_{t \geq 0} \stackrel{\mathcal{D}}{=}\left(\sigma B_{t}\right)_{t \geq 0}$, we have that

$$
\left(L^{\sigma}(T, x)\right)_{x \in \mathbb{R}} \stackrel{\mathcal{D}}{=}\left(\frac{1}{\sigma} L\left(T, \frac{x}{\sigma}\right)\right)_{x \in \mathbb{R}}, \quad \widehat{H}_{T}^{\sigma} \stackrel{\mathcal{D}}{=} \frac{1}{\sigma} \widehat{H}_{T}, \quad \sigma, T>0 .
$$

\footnotetext{
${ }^{6}$ Indeed, the projections of $S^{(1)}$ and $S^{(2)}$ onto the lines with slope 1 and -1 in $\mathbb{R}^{2}$, respectively, are two independent copies of one-dimensional simple random walk on $\sqrt{2} \mathbb{Z}$.

${ }^{7}$ In fact, BS95, Theorem 1.3] applies only to simple random walk, but an inspection of its proof reveals that it in fact holds in the generality of our setting.
} 
II. The second invariance principle we will rely on was shown in [A86, Theorem 1.8], and states that

$$
\left(\sigma^{-\frac{4}{3}}\left(S_{\left\lfloor\sigma^{\frac{2}{3}} t\right]}\right)_{t \in[0, T]}, \mathbb{1}_{\left\{H_{\left\lfloor\sigma^{\frac{2}{3}} T\right\rfloor}=0\right\}}\right) \stackrel{\sigma \rightarrow \infty}{\Longrightarrow}\left(\left(B_{t}\right)_{t \in[0, T]}, \mathbb{1}_{\{U>T\}}\right), \quad T>0
$$

where the law of the random variable $U$ is given by its conditional distribution given the underlying Brownian motion as

$$
\widehat{P}\left(U>T \mid\left(B_{t}\right)_{t \in[0, T]}\right)=e^{-\widehat{H}_{T}},
$$

and the limit $\sigma \rightarrow \infty$ is to be taken subject to the following three technical restrictions:

$$
\begin{aligned}
& \text { (a) } \lim _{N \rightarrow \infty} \limsup _{\sigma \rightarrow \infty} E\left(\left(S_{1} / \sigma\right)^{2} \mathbb{1}_{\left\{\left|S_{1} / \sigma\right|>N\right\}}\right)=0 ; \\
& \text { (b) } \lim _{\sigma \rightarrow \infty} \sigma^{\frac{2}{3}} \max _{x \in \mathbb{Z}} P\left(S_{1}=x\right)=0 ; \\
& \text { (c) } \min _{\sigma \geq 1} \min _{0<|x| \leq c_{1} \sigma} \sigma P\left(S_{1}=x\right) \geq c_{2} \text { for some } c_{1}, c_{2}>0 .
\end{aligned}
$$

The analogue of (2.19) for $\sigma \rightarrow \infty$ under (2.23) is not known. Therefore, on top of (2.23), we will require a uniform exponential moment for $S_{1} / \sigma$, i.e.,

$$
\sup _{\sigma \geq 1} E\left(e^{\varepsilon\left|S_{1}\right| / \sigma}\right)<\infty \quad \text { for some } \varepsilon>0,
$$

which is obviously stronger than $(2.23)$ (a) and replaces the second condition in (1.1). Note that the random walk with $P\left(S_{1}=x\right)=\frac{1}{2 L}$ for $x \in\{-L, \ldots,-1,1, \ldots, L\}$ satisfies 2.23)-(2.24) (for which $\sigma^{2} \sim L^{2} / 3$ ). So does the random walk with $P\left(S_{1}=x\right)=\frac{1}{2 L}\left(\frac{L-1}{L}\right)^{|x|-1}$ for $x \in \mathbb{Z} \backslash\{0\}$ (for which $\left.\sigma^{2} \sim L^{2}\right)$.

\section{LARGe DEViations}

To prove the results in Sections 2.1 2.3, we will actually prove something much stronger, namely, scaling of the large deviation rate function for the empirical drift of the path. We will show that the rate function for the Domb-Joyce model and its variants scales to the rate function for the Edwards model. Now, the existence of the rate function for the Domb-Joyce model has been established only in a rather limited number of cases, namely, under the assumption in (1.6). In Section 3.1 we summarize what is known for this special case. For the variants of the Domb-Joyce model the existence is still open. Therefore we will have to work with liminf's and limsup's. The existence of the rate function for the Edwards model has been proved in our recent paper vdHdHK02 and its properties will be described in Section 3.2. Another important object is the cumulant generating function for the Edwards model, which will be introduced in Section 3.3. More refined large deviation properties for the Edwards model also proved in vdHdHK02, which will be needed in our proofs, are presented in Section 3.4.

\subsection{Large deviations for the Domb-Joyce model.}

Throughout this section we assume (1.6). The main object of interest in this section is the rate function $I_{\beta}$ defined by

$$
I_{\beta}(\theta)=-\lim _{n \rightarrow \infty} \frac{1}{n} \log E\left(e^{-\beta H_{n}} \mathbb{1}_{\left\{S_{n} \approx \theta n\right\}}\right)=-\lim _{n \rightarrow \infty} \frac{1}{n} \log \left\{Z_{n}^{\beta} Q_{n}^{\beta}\left(S_{n} \approx \theta n\right)\right\}, \quad \theta \in \mathbb{R},
$$

where $S_{n} \approx \theta n$ means that either $S_{n}=\lfloor\theta n\rfloor$ or $S_{n}=\lceil\theta n\rceil$ (possibly depending on the parity of these numbers). For $\beta=\infty$ we adopt the convention $e^{-\infty H_{n}}=\mathbb{1}_{\left\{H_{n}=0\right\}}$. Obviously, $I_{\beta}(\theta)=I_{\beta}(-\theta)$, and $I_{\beta}(\theta)=\infty$ when $\theta>L$. Therefore we may restrict ourselves to $\theta \in[0, L]$.

\footnotetext{
${ }^{8}$ In fact, $I_{\beta}$ differs by a constant from what is usually called a rate function: $I_{\beta}-r^{*}$ is the true rate function (see Theorem 1.1(ii)).
} 
Recall the three quantities $r^{*}, \theta^{*}, \sigma^{*}$ in Theorem 1.1. In the next theorem a fourth quantity $\theta^{* *}$ appears, which, like the others, depends on $\beta$ and on the distribution of $S_{1}$.

Theorem 3.1 (LDP). Fix $\beta \in[0, \infty]$, assume (1.6), and exclude the trivial case $(\beta, L)=(\infty, 1)$.

(i) For any $\theta \in[0, L]$, the limit $I_{\beta}(\theta)$ in (3.1) exists and is finite.

(ii) $I_{\beta}$ is continuous and convex on $[0, L]$, and continuously differentiable on $(0, L)$.

(iii) There is a number $\theta^{* *} \in\left(0, \theta^{*}\right)$ such that $I_{\beta}$ is linearly decreasing on $\left[0, \theta^{* *}\right]$, real-analytic and strictly convex on $\left(\theta^{* *}, L\right)$, and attains its unique minimum at $\theta^{*}$ with height $I_{\beta}\left(\theta^{*}\right)=r^{*}$ and curvature $I_{\beta}^{\prime \prime}\left(\theta^{*}\right)=1 / \sigma^{* 2}$.

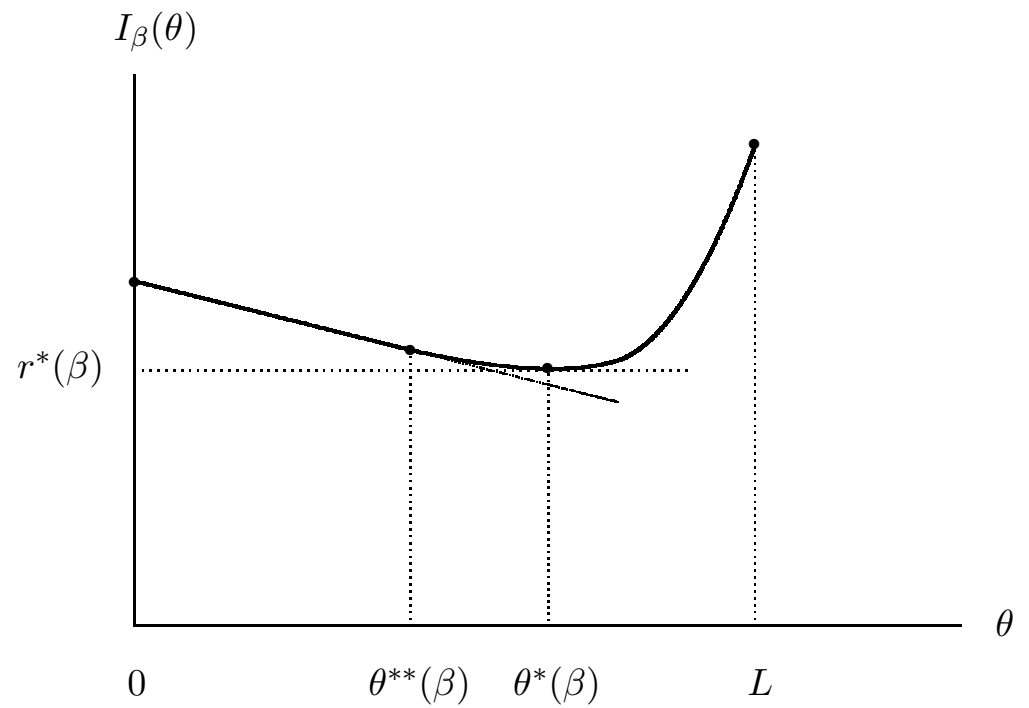

Fig. 1. Qualitative picture of $\theta \mapsto I_{\beta}(\theta)$.

Theorem 3.1 is proved for simple random walk $(L=1)$ in dH00, Theorem IX.32], relying on the methods and results of GH93]. We have checked that this proof can be extended to general $L \in \mathbb{N}$ with the help of the methods and results of [K94.

The main ingredients of the proof of Theorem 3.1 are reflection arguments and precise analytic knowledge of the contribution to the intersection local time coming from paths that satisfy the socalled "bridge condition", i.e., lie between their starting and ending locations $S_{0}$ and $S_{n}$. The linear piece of the rate function has the following intuitive explanation. If $\theta \geq \theta^{* *}$, then the optimal strategy for the path to realize $S_{n} \approx \theta n$ is to assume local drift $\theta$ during $n$ steps. In particular, the path then satisfies the bridge condition, and this reasoning leads to the strict convexity and real-analyticity of the rate function on $\left(\theta^{* *}, L\right)$. If, on the other hand, $0 \leq \theta<\theta^{* *}$, then this strategy is too expensive, since too small a drift leads to too many self-intersections. Therefore the optimal strategy now is to move with local drift $\theta^{* *}$ during $\frac{\theta^{* *}+\theta}{2 \theta^{* *}} n$ steps and with local drift $-\theta^{* *}$ during the remaining $\frac{\theta^{* *}-\theta}{2 \theta^{* *}} n$ steps, thus making an overshoot of size $\frac{\theta^{* *}-\theta}{2} n$, and this reasoning leads to the linearity of the rate function on $\left[0, \theta^{* *}\right]$.

\subsection{Large deviations for the Edwards model.}

The analogue of (3.1) for the Edwards model is the rate function $\widehat{I}_{\beta}$ defined by

$$
\widehat{I}_{\beta}(b)=-\lim _{T \rightarrow \infty} \frac{1}{T} \log \widehat{E}\left(e^{-\beta \widehat{H}_{T}} \mathbb{1}_{\left\{B_{T} \approx b T\right\}}\right)=-\lim _{T \rightarrow \infty} \frac{1}{T} \log \left\{\widehat{Z}_{T}^{\beta} \widehat{Q}_{T}^{\beta}\left(B_{T} \approx b T\right)\right\}, \quad b \in \mathbb{R},
$$


where $B_{T} \approx b T$ means that $\left|B_{T}-b T\right| \leq \gamma_{T}$ for some $\gamma_{T}>0$ such that $\gamma_{T} / T \rightarrow 0$ and $\gamma_{T} / \sqrt{T} \rightarrow \infty$ as $T \rightarrow \infty$. In vdHdHK02 we proved that the limit in (3.2) exists and is independent of the choice of $\gamma_{T}$. From (1.10) it is clear that this rate function satisfies the scaling relation

$$
\beta^{-\frac{2}{3}} \widehat{I}_{\beta}\left(\beta^{\frac{1}{3}} \cdot\right)=\widehat{I}_{1}(\cdot),
$$

provided the limit in (3.2) exists for $\beta=1$.

Recall the three quantities $a^{*}, b^{*}, c^{*}$ in Theorem 1.2. In the next theorem a fourth quantity $b^{* *}$ appears.

Theorem $3.2(\mathrm{LDP})$.

(i) For any $b \in[0, \infty)$, the limit $\widehat{I}_{1}(b)$ in (3.2) exists and is finite (and is independent of the choice of $\left.\gamma_{T}\right)$.

(ii) $\widehat{I}_{1}$ is continuous and convex on $[0, \infty)$, and continuously differentiable on $(0, \infty)$.

(iii) There is a number $b^{* *} \in\left(0, b^{*}\right)$ such that $\widehat{I}_{1}$ is linearly decreasing on $\left[0, b^{* *}\right]$, real-analytic and strictly convex on $\left(b^{* *}, \infty\right)$, and attains its unique minimum at $b^{*}$ with height $\widehat{I}_{1}\left(b^{*}\right)=a^{*}$ and curvature $\widehat{I}_{1}^{\prime \prime}\left(b^{*}\right)=1 / c^{* 2}$.

Theorem 3.2 is proved in vdHdHK02]. The numerical value of $b^{* *}$ is $b^{* *} \approx 0.85$. Note the close analogy with Theorem 3.1. The linear piece has the same intuitive explanation in terms of overshoot.

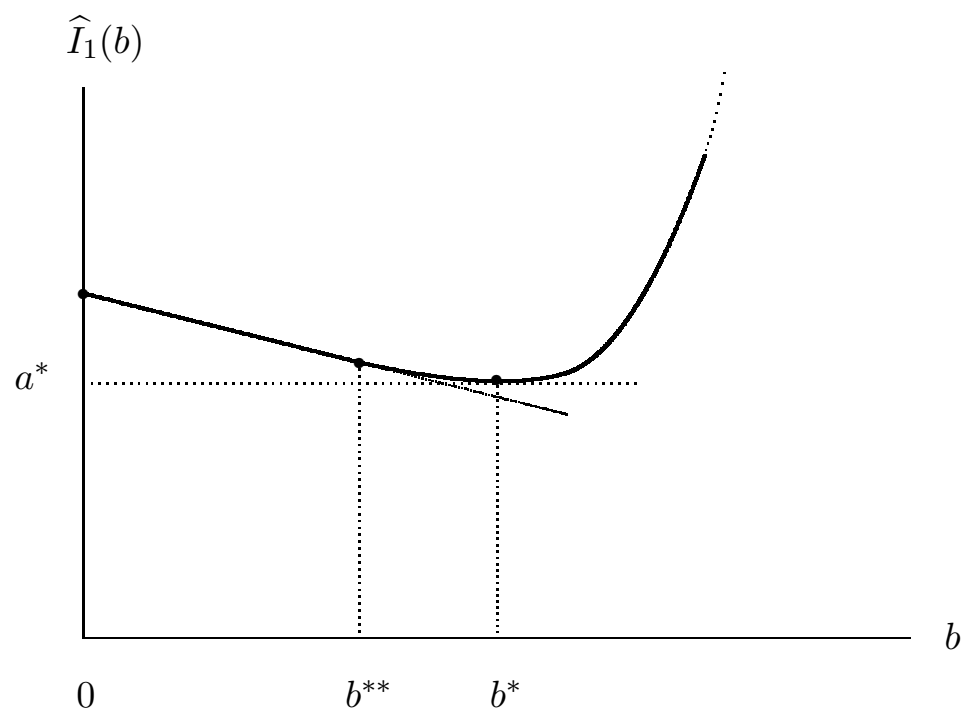

Fig. 2. Qualitative picture of $b \mapsto \widehat{I}_{1}(b)$.

Denote by $\widehat{I}_{\beta}^{\sigma}$ the rate function in $(3.2)$ for the Brownian motion with generator $\frac{1}{2} \sigma^{2} \Delta$. Like $\widehat{I}_{\beta}$, it satisfies the scaling relation $\beta^{-\frac{2}{3}} \widehat{I}_{\beta}^{\sigma}\left(\beta^{\frac{1}{3}} \cdot\right)=\widehat{I}_{1}^{\sigma}(\cdot)$ in $(3.3)$. Furthermore, from (2.20) we obtain the scaling relation

$$
\widehat{I}_{1}^{\sigma}(\cdot)=\sigma^{-\frac{2}{3}} \widehat{I}_{1}\left(\sigma^{-\frac{2}{3}} \cdot\right)
$$




\subsection{Cumulant generating function for the Edwards model.}

There is an intimate connection between the rate function in (3.2) and the cumulant generating function $\Lambda^{+}: \mathbb{R} \rightarrow \mathbb{R}$ given by

$$
\Lambda^{+}(\mu)=\lim _{T \rightarrow \infty} \frac{1}{T} \log \widehat{E}\left(e^{-\widehat{H}_{T}} e^{\mu B_{T}} \mathbb{1}_{\left\{B_{T} \geq 0\right\}}\right), \quad \mu \in \mathbb{R} .
$$

Proposition 3.3 (Exponential moments).

(i) For any $\mu \in \mathbb{R}$, the limit $\Lambda^{+}(\mu)$ in (3.5) exists and is finite.

(ii) There is a number $\rho\left(a^{* *}\right)>0$ such that $\Lambda^{+}$is constant on $\left(-\infty,-\rho\left(a^{* *}\right)\right]$, and strictly increasing, strictly convex and real-analytic on $\left(-\rho\left(a^{* *}\right), \infty\right)$. In $-\rho\left(a^{* *}\right), \Lambda^{+}$is continuous, but not differentiable.

(iii) $\lim _{\mu \downarrow-\rho\left(a^{* *}\right)}\left(\Lambda^{+}\right)^{\prime}(\mu)=b^{* *},\left(\Lambda^{+}\right)^{\prime}(0)=b^{*}$, and $\lim _{\mu \rightarrow \infty}\left(\Lambda^{+}\right)^{\prime}(\mu)=\infty$.

(iv) The restriction of $\widehat{I}_{1}$ to $[0, \infty)$ is the Legendre transform of $\Lambda^{+}$, i.e.,

$$
\widehat{I}_{1}(b)=\max _{\mu \in \mathbb{R}}\left[\mu b-\Lambda^{+}(\mu)\right], \quad b \geq 0 .
$$

Proposition 3.3 is proved in vdHdHK02. The numerical value of $\rho\left(a^{* *}\right)$ is $\rho\left(a^{* *}\right) \approx 0.78$. By $(3.6)$, $-\rho\left(a^{* *}\right)$ is the slope of the linear piece in Fig. 2. Note that $\Lambda^{+}(0)=-a^{*}$ by Theorem 1.2(ii) and (3.3).

As a consequence of Proposition 3.3(ii), the maximum on the right-hand side of (3.6) is attained in some $\mu>-\rho\left(a^{* *}\right)$ if $b>b^{* *}$ and in $\mu=-\rho\left(a^{* *}\right)$ if $0 \leq b \leq b^{* *}$.

Let $\Lambda^{-}$denote the cumulant generating function with $\mathbb{1}_{\left\{B_{T} \leq 0\right\}}$ instead of $\mathbb{1}_{\left\{B_{T} \geq 0\right\}}$. Then analogous assertions for $\Lambda^{-}$hold as well. In particular, the restriction of $\widehat{I}_{1}$ to $(-\infty, 0]$ is the Legendre transform of $\Lambda^{-}$. By symmetry, $\Lambda^{+}(-\mu)=\Lambda^{-}(\mu)$ for any $\mu \in \mathbb{R}$. Consequently, the cumulant generating function $\Lambda(\mu)=\lim _{T \rightarrow \infty} \frac{1}{T} \log \widehat{E}\left(e^{-\widehat{H}_{T}} e^{\mu B_{T}}\right)=\Lambda^{+}(\mu) \vee \Lambda^{-}(\mu)=\Lambda^{+}(|\mu|)$ exists for any $\mu \in \mathbb{R}$ and is not differentiable at 0 .

Let $\Lambda_{\sigma}^{+}$and $\Lambda_{\sigma}^{-}$denote the corresponding cumulant generating functions for the Edwards model with variance $\sigma^{2}$ (i.e., where the generator of the underlying Brownian motion is $\frac{1}{2} \sigma^{2} \Delta$ ). Then we have the scaling relation $\sigma^{\frac{2}{3}} \Lambda_{\sigma}^{+}\left(\sigma^{-\frac{4}{3}} \cdot\right)=\Lambda^{+}(\cdot)$. Moreover, we have

$$
\widehat{I}_{1}^{\sigma}(b)=\max _{\mu \in \mathbb{R}}\left[\mu b-\Lambda_{\sigma}^{+}(\mu)\right]= \begin{cases}\max _{\mu \geq 0}\left[\mu b-\Lambda_{\sigma}^{+}(\mu)\right] & \text { if } b \geq b^{*} \sigma^{\frac{2}{3}}, \\ \max _{\mu \leq 0}\left[\mu b-\Lambda_{\sigma}^{+}(\mu)\right] & \text { if } 0 \leq b \leq b^{*} \sigma^{\frac{2}{3}} .\end{cases}
$$

Analogous assertions hold for $\Lambda_{\sigma}^{-}$.

\subsection{More refined large deviation properties for the Edwards model.}

In the proofs we will need some further refinements of Proposition 3.3. Abbreviate $B_{[0, T]}=$ $\left(B_{t}\right)_{t \in[0, T]}$. For $T>0, \delta, C \in(0, \infty]$ and $\alpha \in[0, \infty)$, define events

$$
\begin{aligned}
\widehat{\mathcal{E}}(\delta, T) & =\left\{B_{[0, T]} \subset\left[-\delta, B_{T}+\delta\right]\right\}, \\
\widehat{\mathcal{E}}^{\leq}(\delta, C ; T) & =\left\{\max _{x \in[-\delta, \delta]} L(T, x) \leq C, \max _{x \in\left[B_{T}-\delta, B_{T}+\delta\right]} L(T, x) \leq C\right\}, \\
\widehat{\mathcal{E}} \geq(\delta, \alpha ; T) & =\left\{\max _{x \in\left[B_{T}-\delta, B_{T}+\delta\right]} L(T, x) \geq \alpha \delta^{-\frac{1}{2}}\right\} .
\end{aligned}
$$

Note that $\widehat{\mathcal{E}}^{\leq}(\delta, \infty ; T)$ and $\widehat{\mathcal{E}}^{\geq}(\delta, 0 ; T)$ are the full space. 
Proposition 3.4 (Overshoots). Fix $\mu>-\rho\left(a^{* *}\right)$. Then:

(i) For any $\delta, C \in(0, \infty]$ there exists a $K_{1}(\delta, C) \in(0, \infty)$ such that

$$
e^{-\Lambda^{+}(\mu) T} \widehat{E}\left(e^{-\widehat{H}_{T}} e^{\mu B_{T}} \mathbb{1}_{\widehat{\mathcal{E}}(\delta, T)} \mathbb{1}_{\widehat{\mathcal{E}} \leq(\delta, C ; T)} \mathbb{1}_{\left\{B_{T} \geq 0\right\}}\right)=K_{1}(\delta, C)+o(1), \quad T \rightarrow \infty .
$$

Moreover, if $\mu=\mu_{b}$ solves $\widehat{I}_{1}(b)=\mu b-\Lambda^{+}(\mu)$, then the same is true when $\mathbb{1}_{\left\{B_{T} \geq 0\right\}}$ is replaced by $\mathbb{1}_{\left\{B_{T} \approx b T\right\}}$.

(ii) For any $\delta, \alpha \in(0, \infty)$ there exists a $K_{2}(\delta, \alpha) \in(0, \infty)$ such that

$$
e^{-\Lambda^{+}(\mu) T} \widehat{E}\left(e^{-\widehat{H}_{T}} e^{\mu B_{T}} \mathbb{1}_{\widehat{\mathcal{E}}(\delta, T)} \mathbb{1}_{\widehat{\mathcal{E}} \geq(\delta, \alpha ; T)} \mathbb{1}_{\left\{B_{T} \geq 0\right\}}\right)=K_{2}(\delta, \alpha)+o(1), \quad T \rightarrow \infty .
$$

(iii) For any $\alpha \in(0, \infty)$,

$$
\lim _{\delta \downarrow 0} \frac{K_{2}(\delta, \alpha)}{K_{1}(\delta, \infty)}=0
$$

Proposition 3.4 is proved in $\mathrm{vdHdHK02}$.

\section{Proof of Theorem 2.1(I)}

In this section we consider the limit $\beta \downarrow 0$. Let $\left(S_{n}\right)_{n \in \mathbb{N}_{0}}$ be a random walk satisfying (1.1). As announced at the beginning of Section 3, we will identify the scaling limit of the entire large deviation rate function (for the linear asymptotics of the endpoint) for the Domb-Joyce model in terms of that for the Edwards model, and we will deduce Theorem 2.1(i) from this scaling limit. However, as pointed out at the beginning of Section 3, the existence of the rate function has not been established in full generality for the Domb-Joyce model, and we will make no attempt to do so. Instead, we will be working with approximative rate functions, which are defined as a limsup or a liminf instead of a lim.

\subsection{Approximative large deviations.}

It will be sufficient to deal with the event $\left\{S_{n} \geq \theta n\right\}$ for $\theta$ to the right of the scaled minimum point of the limiting rate function, and with $\left\{S_{n} \leq \theta n\right\}$ for $\theta$ to the left of it. To this end, define

$$
I_{\beta}^{+}(\theta ; \widetilde{\theta})= \begin{cases}-\liminf _{n \rightarrow \infty} \frac{1}{n} \log E\left(e^{-\beta H_{n}} \mathbb{1}_{\left\{S_{n} \geq \theta n\right\}}\right) & \text { if } \theta \geq \widetilde{\theta}, \\ -\liminf _{n \rightarrow \infty} \frac{1}{n} \log E\left(e^{-\beta H_{n}} \mathbb{1}_{\left\{0 \leq S_{n} \leq \theta n\right\}}\right) & \text { if } \theta \leq \widetilde{\theta},\end{cases}
$$

and define $I_{\beta}^{-}(\theta ; \widetilde{\theta})$ in the same way with $\lim \sup$ instead of $\lim$ inf. For $\beta=\infty$, recall the convention $e^{-\infty H_{n}}=\mathbb{1}_{\left\{H_{n}=0\right\}}$.

In the special case of (1.6), we know from Theorem 3.1 that the limit $I_{\beta}(\theta)$ in (3.1) exists. Since $I_{\beta}$ is unimodal with unique minimiser $\theta^{*}$, it follows that both limits in (4.1) exist and that

$$
I_{\beta}^{+}\left(\theta ; \theta^{*}\right)=I_{\beta}^{-}\left(\theta ; \theta^{*}\right)=I_{\beta}(\theta), \quad 0 \leq \theta \leq L .
$$

Our main result in this section shows that the approximative rate function in (4.1) scales, as $\beta \downarrow 0$, to the rate function for the Edwards model with parameter $\sigma$.

Proposition 4.1. Fix $\sigma \in(0, \infty)$. Then, under (1.1),

$$
\begin{aligned}
& \liminf _{\beta \downarrow 0} \beta^{-\frac{2}{3}} I_{\beta}^{-}\left(b \beta^{\frac{1}{3}} ; b^{*} \beta^{\frac{1}{3}} \sigma^{\frac{2}{3}}\right) \geq \widehat{I}_{1}^{\sigma}(b), \quad b \geq 0, \\
& \limsup _{\beta \downarrow 0} \beta^{-\frac{2}{3}} I_{\beta}^{+}\left(b \beta^{\frac{1}{3}} ; b^{*} \beta^{\frac{1}{3}} \sigma^{\frac{2}{3}}\right) \leq \widehat{I}_{1}^{\sigma}(b), \quad b>b^{* *} \sigma^{\frac{2}{3}} .
\end{aligned}
$$


Proposition 4.1 is proved in Section 4.3. In the special case of (1.6), we infer from Theorem 3.1 and Proposition that

$$
\lim _{\beta \downarrow 0} \beta^{-\frac{2}{3}} I_{\beta}\left(b \beta^{\frac{1}{3}}\right)=\widehat{I}_{1}^{\sigma}(b), \quad b>b^{* *} \sigma^{\frac{2}{3}} .
$$

The proof of (4.4) for $0 \leq b \leq b^{* *} \sigma^{\frac{2}{3}}$ remains open. To extend (4.4) to this regime would require some further refinements of our method (see Section 7).

\subsection{Proof of Theorem 2.1(i) and Corollary 2.2.}

1. Fix $\varepsilon>0$. We will show that, for $\beta>0$ sufficiently small,

$$
\lim _{n \rightarrow \infty} \frac{1}{n} \log Q_{n}^{\beta}\left(\frac{\left|S_{n}\right|}{\beta^{\frac{1}{3}} n}-b^{*} \sigma^{\frac{2}{3}}>\varepsilon\right)<0 .
$$

This obviously implies the upper half of the statement in (2.1). The lower half can be derived in the same manner.

2. To prove (4.6), put $b^{\prime}=b^{*} \sigma^{\frac{2}{3}}+\frac{\varepsilon}{2}$ and $b=b^{*} \sigma^{\frac{2}{3}}+\varepsilon$. Since $\widehat{I}_{1}^{\sigma}$ is strictly increasing on $\left[b^{*} \sigma^{\frac{2}{3}}, \infty\right)$, it is possible to pick $\gamma>0$ so small (depending on $\varepsilon$ ) that

$$
\widehat{I}_{1}^{\sigma}(b)-\widehat{I}_{1}^{\sigma}\left(b^{\prime}\right)-2 \gamma>0 .
$$

According to Proposition 4.1, we may pick $\beta>0$ so small (depending on $\gamma$ ) that

$$
I_{\beta}^{-}\left(b \beta^{\frac{1}{3}} ; b^{*} \beta^{\frac{1}{3}} \sigma^{\frac{2}{3}}\right) \geq\left[\widehat{I}_{1}^{\sigma}(b)-\gamma\right] \beta^{\frac{2}{3}}, \quad I_{\beta}^{+}\left(b^{\prime} \beta^{\frac{1}{3}} ; b^{*} \beta^{\frac{1}{3}} \sigma^{\frac{2}{3}}\right) \leq\left[\widehat{I}_{1}^{\sigma}\left(b^{\prime}\right)+\gamma\right] \beta^{\frac{2}{3}} .
$$

Now we can bound (recall (1.3))

$$
\begin{aligned}
Q_{n}^{\beta}\left(\frac{S_{n}}{\beta^{\frac{1}{3}} n}-b^{*} \sigma^{\frac{2}{3}}>\varepsilon\right) & =\frac{E\left(e^{-\beta H_{n}} \mathbb{1}_{\left\{S_{n}>b \beta^{\frac{1}{3}} n\right\}}\right)}{E\left(e^{-\beta H_{n}}\right)} \leq \frac{E\left(e^{-\beta H_{n}} \mathbb{1}_{\left\{S_{n}>b \beta^{\frac{1}{3}} n\right\}}\right)}{E\left(e^{-\beta H_{n}} \mathbb{1}_{\left\{S_{n}>b^{\prime} \beta^{\frac{1}{3}} n\right\}}\right)} \\
& \leq \exp \left\{-n\left[I_{\beta}^{-}\left(b \beta^{\frac{1}{3}} ; b^{*} \beta^{\frac{1}{3}} \sigma^{\frac{2}{3}}\right)-I_{\beta}^{+}\left(b^{\prime} \beta^{\frac{1}{3}} ; b^{*} \beta^{\frac{1}{3}} \sigma^{\frac{2}{3}}\right)\right]+o(n)\right\},
\end{aligned}
$$

where we use the definitions of $I_{\beta}^{-}$and $I_{\beta}^{+}$. Insert (4.7)-(4.8), to see that the term between square brackets in the exponent of (4.9) is strictly positive. This implies (4.6).

3. The proof of Corollary 2.2 is as follows. Assume (1.6). First, by (4.5), the function $f_{\beta}$ defined by $f_{\beta}(\cdot)=\beta^{-\frac{2}{3}} I_{\beta}\left(\beta^{\frac{1}{3}}.\right)$ converges to $\widehat{I}_{1}^{\sigma}$ on $\left(b^{* *} \sigma^{\frac{2}{3}}, \infty\right)$. In particular, the unique minimal value of $f_{\beta}$, which is $r^{*}(\beta) \beta^{-\frac{2}{3}}$ by Theorem 3.1, converges to the unique minimal value of $\widehat{I}_{1}^{\sigma}$, which is $a^{*} \sigma^{-\frac{2}{3}}$ by Theorem 3.2. This proves the first assertion in (2.3). Next, by (4.5), $f_{\beta}$ converges to $\widehat{I}_{1}^{\sigma}$ in the three points $b^{*} \sigma^{\frac{2}{3}}-\varepsilon, b^{*} \sigma^{\frac{2}{3}}$ and $b^{*} \sigma^{\frac{2}{3}}+\varepsilon$ for $\varepsilon>0$ small enough. For $\beta$ small enough, both $f_{\beta}\left(b^{*} \sigma^{\frac{2}{3}}-\varepsilon\right)$ and $f_{\beta}\left(b^{*} \sigma^{\frac{2}{3}}+\varepsilon\right)$ are strictly larger than $f_{\beta}\left(b^{*} \sigma^{\frac{2}{3}}\right)$. By unimodality, this implies that the unique minimiser of $f_{\beta}$, which is $\theta^{*}(\beta) \beta^{-\frac{1}{3}}$ by Theorem 3.1, lies in $\left(b^{*} \sigma^{\frac{2}{3}}-\varepsilon, b^{*} \sigma^{\frac{2}{3}}+\varepsilon\right)$. Let $\varepsilon \downarrow 0$ to obtain the second assertion in (2.3).

Note that convexity of $f_{\beta}$ yields that even $\left(f_{\beta}\right)^{\prime}$ converges to $\left(\widehat{I}_{1}^{\sigma}\right)^{\prime}$. However, we have no control over $\left(f_{\beta}\right)^{\prime \prime}$, which is why we are unable to prove (2.5).

\subsection{Proof of Proposition 4.1.}

In Section 4.3.1 we prove (4.3), in Section 4.3.2 we prove (4.4). The main idea is to cut the path into smaller pieces to which the weak convergence assertion in 2.18 can be applied. The mutual interaction between the pieces has to be controlled appropriately. This is done by providing estimates 
in which either the pieces are independent or there is an interaction only between neighboring pieces. We define

$$
H_{n}^{\prime}=\sum_{\substack{i, j=1 \\ i \neq j}}^{n} \mathbb{1}_{\left\{S_{i}=S_{j}\right\}}=H_{n}-2\left(\ell_{n}(0)-1\right) .
$$

The proof runs via the moment generating function

$$
Z_{n}^{\beta}(\mu)=E\left(e^{-\beta H_{n}^{\prime}} e^{\mu \beta^{\frac{1}{3}} S_{n}}\right), \quad n \in \mathbb{N}, \mu \in \mathbb{R}
$$

which is the discrete analogue of the expectation in (3.5).

\subsubsection{Proof of (4.3).}

1. Fix $b \geq b^{*} \sigma^{\frac{2}{3}}$. Use the exponential Chebyshev inequality to get the following upper bound for $\mu \geq 0$ :

$$
E\left(e^{-\beta H_{n}} \mathbb{1}_{\left\{S_{n} \geq b \beta^{\frac{1}{3}} n\right\}}\right) \leq e^{-\mu b \beta^{\frac{2}{3}} n} Z_{n}^{\beta}(\mu) .
$$

Fix a large auxiliary parameter $T>0$ and abbreviate $T_{\beta}=\beta^{-\frac{2}{3}} T$. Split the path of length $n$ into $n / T_{\beta}$ pieces of length $T_{\beta}$. (To simplify the notation, assume that both $n / T_{\beta}$ and $T_{\beta}$ are integers.) Drop the interaction between any two of the pieces, to obtain an upper bound on $Z_{n}^{\beta}(\mu)$. After the pieces are decoupled they are independent of each other. This reasoning yields

$$
Z_{n}^{\beta}(\mu) \leq\left(Z_{T_{\beta}}^{\beta}(\mu)\right)^{n / T_{\beta}} .
$$

Substitute this estimate into (4.12), take logs, divide by $\beta^{\frac{2}{3}} n$ and let $n \rightarrow \infty$, to obtain (recall (4.1))

$$
\begin{aligned}
\beta^{-\frac{2}{3}} I_{\beta}^{-}\left(b \beta^{\frac{1}{3}} ; b^{*} \beta^{\frac{1}{3}} \sigma^{\frac{2}{3}}\right) & \geq-\beta^{-\frac{2}{3}} \liminf _{n \rightarrow \infty} \frac{1}{n} \log (\text { l.h.s. of }(4.12)) \\
& \geq-\beta^{-\frac{2}{3}} \liminf _{n \rightarrow \infty} \frac{1}{n} \log \left[e^{-\mu b \beta^{\frac{2}{3}}} n\left(Z_{T_{\beta}}^{\beta}(\mu)\right)^{n \beta^{\frac{2}{3}} / T}\right] \\
& =\mu b-\frac{1}{T} \log Z_{T_{\beta}}^{\beta}(\mu) .
\end{aligned}
$$

2. The next lemma states that, under (1.1), the expectation in the right-hand side of (4.14) converges to the corresponding Brownian expectation. Its proof is given in part 4.

Lemma 4.2. Assume (1.1). Then, for any $\mu \in \mathbb{R}$,

$$
\lim _{\beta \downarrow 0} Z_{T_{\beta}}^{\beta}(\mu)=\widehat{E}\left(e^{-\widehat{H}_{T}^{\sigma}} e^{\mu B_{T}^{\sigma}}\right) .
$$

Lemma 4.2 applied to (4.14) yields

$$
\liminf _{\beta \downarrow 0}\left[\beta^{-\frac{2}{3}} I_{\beta}^{-}\left(b \beta^{\frac{1}{3}} ; b^{*} \beta^{\frac{1}{3}} \sigma^{\frac{2}{3}}\right)\right] \geq \mu b-\frac{1}{T} \log \widehat{E}\left(e^{-\widehat{H}_{T}^{\sigma}} e^{\mu B_{T}^{\sigma}}\right), \quad \mu \geq 0 .
$$

Now let $T \rightarrow \infty$ and use (3.5), to obtain

$$
\liminf _{\beta \downarrow 0}\left[\beta^{-\frac{2}{3}} I_{\beta}^{-}\left(b \beta^{\frac{1}{3}} ; b^{*} \beta^{\frac{1}{3}} \sigma^{\frac{2}{3}}\right)\right] \geq \mu b-\Lambda_{\sigma}^{+}(\mu) .
$$

Maximize over $\mu \geq 0$ and use (3.7), to arrive at the assertion in (4.3).

3. The proof for $0 \leq b \leq b^{*} \sigma^{\frac{2}{3}}$ follows the same pattern. Estimate, for $\mu \leq 0$,

$$
E\left(e^{-\beta H_{n}} \mathbb{1}_{\left\{0 \leq S_{n} \leq b \beta^{\frac{1}{3}} n\right\}}\right) \leq e^{-\mu b \beta^{\frac{2}{3}} n} Z_{n}^{\beta}(\mu) .
$$


In the same way as above we obtain

$$
\liminf _{\beta \downarrow 0}\left[\beta^{-\frac{2}{3}} I_{\beta}^{-}\left(b \beta^{\frac{1}{3}} ; b^{*} \beta^{\frac{1}{3}} \sigma^{\frac{2}{3}}\right)\right] \geq \mu b-\Lambda_{\sigma}^{+}(\mu) .
$$

Now maximize over $\mu \leq 0$ and again use 3.7 .

4. We finish by proving Lemma 4.2.

Proof of Lemma 4.2. Fix $\mu \in \mathbb{R}$. By the weak convergence assertion in (2.18), together with dominated convergence, we have for every $K>0$,

$$
\lim _{\beta \downarrow 0} E\left(e^{-\beta H_{T_{\beta}}^{\prime}} e^{\mu \beta^{\frac{1}{3}} S_{T_{\beta}}} \mathbb{1}_{\left\{\beta^{\frac{1}{3}}\left|S_{T_{\beta}}\right|<K\right\}}\right)=\widehat{E}\left(e^{-\widehat{H}_{T}^{\sigma}} e^{\mu B_{T}^{\sigma}} \mathbb{1}_{\left\{\left|B_{T}^{\sigma}\right|<K\right\}}\right) .
$$

The right-hand side of $(4.20)$ increases to $\widehat{E}\left(e^{-\widehat{H}_{T}^{\sigma}} e^{\mu B_{T}^{\sigma}}\right)$ as $K \rightarrow \infty$. Therefore it suffices to show that

$$
\lim _{K \rightarrow \infty} \limsup _{\beta \downarrow 0} E\left(e^{-\beta H_{T_{\beta}}^{\prime}} e^{\mu \beta^{\frac{1}{3}} S_{T_{\beta}}} \mathbb{1}_{\left\{\beta^{\frac{1}{3}}\left|S_{T_{\beta}}\right| \geq K\right\}}\right)=0 .
$$

To prove (4.21), use the Cauchy-Schwarz inequality:

$$
E\left(e^{-\beta H_{T_{\beta}}^{\prime}} e^{\mu \beta^{\frac{1}{3}} S_{T_{\beta}}} \mathbb{1}_{\left\{\beta^{\frac{1}{3}}\left|S_{T_{\beta}}\right| \geq K\right\}}\right)^{2} \leq P\left(\beta^{\frac{1}{3}}\left|S_{T_{\beta}}\right| \geq K\right) E\left(e^{2 \mu \beta^{\frac{1}{3}} S_{T_{\beta}}}\right) .
$$

The first term converges to $\widehat{P}\left(\left|B_{T}\right| \geq K\right)$ as $\beta \downarrow 0$, which vanishes as $K \rightarrow \infty$. Therefore it suffices to show that

$$
\limsup _{\beta \downarrow 0} E\left(e^{2 \mu \beta^{\frac{1}{3}} S_{T_{\beta}}}\right)<\infty .
$$

To prove (4.23), denote the moment generating function of $S_{1}$ by $\varphi(t)=E\left(e^{t S_{1}}\right), t \in \mathbb{R}$. Then

$$
E\left(e^{2 \mu \beta^{\frac{1}{3}} S_{T_{\beta}}}\right)=\varphi\left(2 \mu \beta^{\frac{1}{3}}\right)^{T_{\beta}} .
$$

By (1.1), the right-hand side is finite for $\beta$ small enough (depending on $\mu$ ). Now note that, by (1.1)-(1.2),

$$
\varphi(t)=1+\frac{1}{2} \sigma^{2} t^{2}+\mathcal{O}\left(|t|^{3}\right), \quad t \rightarrow 0
$$

Put $t=2 \mu \beta^{\frac{1}{3}}$ and combine $\left.(4.24)-4.25\right)$, to get

$$
E\left(e^{2 \mu \beta^{\frac{1}{3}} S_{T_{\beta}}}\right) \leq e^{T_{\beta}\left[\frac{1}{2} \sigma^{2} t^{2}+\mathcal{O}\left(|t|^{3}\right)\right]}=e^{2 \mu^{2} \sigma^{2} T\left[1+\mathcal{O}\left(\beta^{\frac{1}{3}}\right)\right]}, \quad \beta \downarrow 0 .
$$

This proves (4.23) and completes the proof of Lemma 4.2.

\subsubsection{Proof of (4.4).}

We again cut the path into pieces as in Section 4.3.1, but this time we keep control of the interaction between the pieces. Since we are looking for a lower bound on an expectation, we may freely require additional properties of the pieces in such a way that we can control their mutual interaction and still perform the limit $\beta \downarrow 0$.

1. Fix $b \geq b^{*} \sigma^{\frac{2}{3}}$. We require that in each piece the path has speed $\geq b \beta^{\frac{1}{3}}$, does not go too far beyond its starting and ending locations, and has local times in the overlapping areas that are uniformly bounded by a constant. To formulate this precisely, for $i=1, \ldots, n / T_{\beta}$ denote by

$$
S^{(i)}=\left(S_{j}^{(i)}\right)_{j=0}^{T_{\beta}} \quad \text { with } \quad S_{j}^{(i)}=S_{j+(i-1) T_{\beta}}-S_{(i-1) T_{\beta}}
$$


the $i$-th piece shifted such that it starts at the origin, and denote by

$$
\ell^{(i)}(x)=\sum_{j=(i-1) T_{\beta}+1}^{i T_{\beta}} \mathbb{1}_{\left\{S_{j}-S_{(i-1) T_{\beta}}=x\right\}}=\sum_{j=1}^{T_{\beta}} \mathbb{1}_{\left\{S_{j}^{(i)}=x\right\}}, \quad x \in \mathbb{Z},
$$

the local times of the $i$-th piece. Fix two parameters $\delta, C \in(0, \infty)$ and estimate

$$
E\left(e^{-\beta H_{n}} \mathbb{1}_{\left\{S_{n} \geq b \beta^{\frac{1}{3}} n\right\}}\right) \geq E\left(e^{-\beta H_{n}} \prod_{i=1}^{n / T_{\beta}}\left[\mathbb{1}_{\mathcal{E}_{i}(\delta, T, \beta)} \mathbb{1}_{\mathcal{E}_{i}^{\leq}(\delta, C, T, \beta)} \mathbb{1}_{\left\{S_{T_{\beta}}^{(i)} \geq b \beta^{\frac{1}{3}} T_{\beta}\right\}}\right]\right),
$$

where the events $\mathcal{E}_{i}(\delta, T, \beta)$ and $\mathcal{E}_{i}^{\leq}(\delta, T, C, \beta)$ are defined by

$$
\begin{aligned}
\mathcal{E}_{i}(\delta, T, \beta) & =\left\{S^{(i)} \subset\left[-\delta \beta^{-\frac{1}{3}}, S_{T_{\beta}}^{(i)}+\delta \beta^{-\frac{1}{3}}\right]\right\}, \\
\mathcal{E}_{i}^{\leq}(\delta, T, C, \beta) & =\left\{\max _{x:|x| \leq \delta \beta^{-\frac{1}{3}}} \ell^{(i)}(x) \leq C \beta^{-\frac{1}{3}}, \max _{x:\left|x-S_{T_{\beta}}^{(i)}\right| \leq \delta \beta^{-\frac{1}{3}}} \ell^{(i)}(x) \leq C \beta^{-\frac{1}{3}}\right\} .
\end{aligned}
$$

2. Next, assume that $\delta<b T / 2$ (i.e., $\left.\delta \beta^{-\frac{1}{3}}<b \beta^{\frac{1}{3}} T_{\beta} / 2\right)$. Then, on the event $\bigcap_{i=1}^{n / T_{\beta}}\left[\mathcal{E}_{i}(\delta, T, \beta) \cap\right.$ $\left.\mathcal{E}_{i}^{\leq}(\delta, T, C, \beta)\right]$, the following hold: (a) there are no mutual intersections between the pieces unless they are neighbors of each other; (b) the $i$-th and the $(i+1)$-st piece have mutual intersections in an interval of length $2 \delta \beta^{-\frac{1}{3}}$ centered at $S_{i T_{\beta}}$ only; (c) in this interval the local times of the $i$-th and the $(i+1)$-st piece are at most $C \beta^{-\frac{1}{3}}$, so that the interaction between them satisfies

$$
e^{-2 \beta \sum_{x} \ell^{(i)}\left(x+S_{(i-1) T_{\beta}}\right) \ell^{(i+1)}\left(x+S_{i T_{\beta}}\right)} \geq e^{-4 \delta C^{2}} .
$$

Therefore, using (4.10), together with (4.32) and (4.31), yields that on the event $\bigcap_{i=1}^{n / T_{\beta}}\left[\mathcal{E}_{i}(\delta, T, \beta) \cap\right.$ $\left.\mathcal{E}_{i}^{\leq}(\delta, T, C, \beta)\right]$, we have

$$
e^{-\beta H_{n}}=e^{-\beta H_{n}^{\prime}-2 \beta\left(\ell_{n}(0)-1\right)} \geq e^{-2 C \beta^{\frac{2}{3}}} e^{-\beta H_{n}^{\prime}} \geq e^{-2 C \beta^{\frac{2}{3}}} e^{-4 \delta C^{2} n / T_{\beta}} \prod_{i=1}^{n / T_{\beta}} e^{-\beta H_{T_{\beta}}^{\prime}(i)},
$$

where $H_{T_{\beta}}^{\prime}(i)$ denotes $H_{T_{\beta}}^{\prime}$ computed for the $i^{\text {th }}$ walk $S^{(i)}$. We substitute (4.33) into (4.29) and note that, after this is done, the pieces are independent. This reasoning yields

$$
E\left(e^{-\beta H_{n}} \mathbb{1}_{\left\{S_{n} \geq b \beta^{\frac{1}{3}} n\right\}}\right) \geq e^{-4 \delta C^{2} n / T_{\beta}} E\left(e^{-\beta H_{T_{\beta}}^{\prime}} \mathbb{1}_{\mathcal{E}_{1}(\delta, T, \beta)} \mathbb{1}_{\mathcal{E}_{1}^{\leq}(\delta, C, T, \beta)} \mathbb{1}_{\left\{S_{T_{\beta}}^{(1)} \geq b \beta^{\frac{1}{3}} T_{\beta}\right\}}\right)^{n / T_{\beta}} .
$$

3. Next, take logs, multiply by $\beta^{-\frac{2}{3}} / n=T_{\beta} / T n$ and let $n \rightarrow \infty$, to obtain

$$
\beta^{-\frac{2}{3}} I_{\beta}^{+}\left(b \beta^{\frac{1}{3}} ; b^{*} \beta^{\frac{1}{3}} \sigma^{\frac{2}{3}}\right) \leq \frac{4 \delta C^{2}}{T}-\frac{1}{T} \log E\left(e^{-\beta H_{T_{\beta}}^{\prime}} \mathbb{1}_{\mathcal{E}_{1}(\delta, T, \beta)} \mathbb{1}_{\mathcal{E}_{1}^{\leq}(\delta, C, T, \beta)} \mathbb{1}_{\left\{S_{T_{\beta}}^{(1)} \geq b \beta^{\frac{1}{3}} T_{\beta}\right\}}\right) .
$$

Let $\beta \downarrow 0$ and use the weak convergence assertions in (2.18)-(2.19), to obtain

$$
\underset{\beta \downarrow 0}{\limsup }\left[\beta^{-\frac{2}{3}} I_{\beta}^{+}\left(b \beta^{\frac{1}{3}} ; b^{*} \beta^{\frac{1}{3}} \sigma^{\frac{2}{3}}\right)\right] \leq \frac{4 \delta C^{2}}{T}-\frac{1}{T} \log \widehat{E}\left(e^{-\widehat{H}_{T}^{\sigma}} \mathbb{1}_{\widehat{\mathcal{E}}(\delta, T)} \mathbb{1}_{\widehat{\mathcal{E}} \leq(\delta, C, T)} \mathbb{1}_{\left\{B_{T}^{\sigma} \geq b T\right\}}\right),
$$

where the events $\widehat{\mathcal{E}}(\delta, T)$ and $\widehat{\mathcal{E}}^{\leq}(\delta, C, T)$ are defined in (3.8)-(3.9).

4. Finally, observe that $\mathbb{1}_{\left\{B_{T}^{\sigma} \geq b T\right\}} \geq \mathbb{1}_{\left\{B_{T}^{\sigma} \approx b^{\prime} T\right\}}$ for any $b^{\prime}>b$ and $T$ sufficiently large (see below (3.2)). Pick $\mu=\mu_{b^{\prime}}$ with $\mu_{b^{\prime}}$ the maximizer in (3.7), i.e., $\widehat{I}_{1}^{\sigma}\left(b^{\prime}\right)=\mu_{b^{\prime}} b^{\prime}-\Lambda_{\sigma}^{+}\left(\mu_{b^{\prime}}\right)$. Since $b \geq b^{*} \sigma^{\frac{2}{3}}$ and $b^{\prime}>b$, we know that $\mu_{b^{\prime}}>0$ (recall (3.6)). Therefore we may bound

$$
\widehat{E}\left(e^{-\widehat{H}_{T}^{\sigma}} \mathbb{1}_{\widehat{\mathcal{E}}(\delta, T)} \mathbb{1}_{\widehat{\mathcal{E}} \leq(\delta, C, T)} \mathbb{1}_{\left\{B_{T}^{\sigma} \geq b T\right\}}\right) \geq e^{-\mu_{b^{\prime}} b^{\prime} T+o(T)} \widehat{E}\left(e^{-\widehat{H}_{T}^{\sigma}} e^{\mu_{b^{\prime}} B_{T}^{\sigma}} \mathbb{1}_{\widehat{\mathcal{E}}(\delta, T)} \mathbb{1}_{\widehat{\mathcal{E}} \leq(\delta, C, T)} \mathbb{1}_{\left\{B_{T}^{\sigma} \approx b^{\prime} T\right\}}\right) .
$$


Insert (4.37) into (4.36), let $T \rightarrow \infty$ and use Proposition 3.4(i) (for the Brownian motion with variance $\sigma^{2}$ instead of 1 ), to arrive at

$$
\limsup _{\beta \downarrow 0}\left[\beta^{-\frac{2}{3}} I_{\beta}^{+}\left(b \beta^{\frac{1}{3}} ; b^{*} \beta^{\frac{1}{3}} \sigma^{\frac{2}{3}}\right)\right] \leq \mu_{b^{\prime}} b^{\prime}-\Lambda_{\sigma}^{+}\left(\mu_{b^{\prime}}\right)=\widehat{I}_{1}^{\sigma}\left(b^{\prime}\right) .
$$

Let $b^{\prime} \downarrow b$ and use the continuity of $\widehat{I}_{1}^{\sigma}$, to complete the proof of (4.4) for $b \geq b^{*} \sigma^{\frac{2}{3}}$.

5. The proof of (4.4) for $b^{* *} \sigma^{\frac{2}{3}}<b \leq b^{*} \sigma^{\frac{2}{3}}$ is analogous. Indeed, 4.27)-(4.36) give that

$$
\limsup _{\beta \downarrow 0}\left[\beta^{-\frac{2}{3}} I_{\beta}^{+}\left(b \beta^{\frac{1}{3}} ; b^{*} \beta^{\frac{1}{3}} \sigma^{\frac{2}{3}}\right)\right] \leq \frac{4 \delta C^{2}}{T}-\frac{1}{T} \log \widehat{E}\left(e^{-\widehat{H}_{T}^{\sigma}} \mathbb{1}_{\widehat{\mathcal{E}}(\delta, T)} \mathbb{1}_{\widehat{\mathcal{E}} \leq(\delta, C, T)} \mathbb{1}_{\left\{0 \leq B_{T}^{\sigma} \leq b T\right\}}\right),
$$

Complete the proof as in (4.37)-(4.38), via $\mathbb{1}_{\left\{0 \leq B_{T}^{\sigma} \leq b T\right\}} \geq \mathbb{1}_{\left\{B_{T}^{\sigma} \approx b^{\prime} T\right\}}$ for any $b^{\prime}<b$ and $T$ sufficiently large, and $\mu_{b^{\prime}}<0$ for any $b^{\prime}<b$.

\section{Proof of Theorem 2.1(II)}

In this section we consider the limit $\sigma \rightarrow \infty$. Let $\left(S_{n}\right)_{n \in \mathbb{N}_{0}}$ be a random walk satisfying (2.23)-(2.24).

\subsection{Approximative large deviations.}

Recall (3.1) and (4.1). Our main result in this section shows that the approximative rate function in (4.1) scales, as $\sigma \rightarrow \infty$, to the rate function for the Edwards model.

Proposition 5.1. Fix $\beta=\infty$. Then, under (2.23)-(2.24),

$$
\begin{aligned}
\liminf _{\sigma \rightarrow \infty} \sigma^{\frac{2}{3}} I_{\infty}^{-}\left(b \sigma^{\frac{2}{3}} ; b^{*} \sigma^{\frac{2}{3}}\right) & \geq \widehat{I}_{1}(b), \quad b \geq 0, \\
\limsup _{\sigma \rightarrow \infty} \sigma^{\frac{2}{3}} I_{\infty}^{+}\left(b \sigma^{\frac{2}{3}} ; b^{*} \sigma^{\frac{2}{3}}\right) & \leq \widehat{I}_{1}(b), \quad b>b^{* *} .
\end{aligned}
$$

Proposition 5.1 implies Theorem 2.1(ii) and Corollary 2.3 in the same way as Proposition 4.1 implies Theorem 2.1(i) and Corollary 2.2 (see Section 4.1). We leave this for the reader to verify.

In the special case of (1.6), subject to (2.23)-(2.24), we know from Theorem 3.1 that the rate function $I_{\beta}$ in (3.1) exists and so we can infer from Proposition 5.1 that

$$
\lim _{\sigma \rightarrow \infty} \sigma^{\frac{2}{3}} I_{\infty}\left(b \sigma^{\frac{2}{3}}\right)=\widehat{I}_{1}(b), \quad b>b^{* *} .
$$

Again, we leave open the convergence for $0 \leq b \leq b^{* *}$.

\subsection{Proof of Proposition 5.1.}

Like in Section 4.3, we decompose the path into pieces to which an appropriate weak convergence assertion can be applied, which is in this case (2.21). The arguments are similar and again revolve around controlling the interaction between neighboring pieces. However, it turns out to be more difficult to handle the mutual avoidance of neighboring pieces than to handle their mutual intersection local times as in Section 4.3. In order to overcome this problem, we use a technique that is reminiscent of the so-called "lace expansion". Throughout the sequel we write " $\left(S_{i}\right)_{i=0}^{n}$ is SAW" if $S_{i} \neq S_{j}$ for all $0 \leq i<j \leq n$.

\subsubsection{Proof of (5.1).}

1. Fix $b \geq b^{*}$ and recall that

$$
I_{\infty}^{+}\left(b \sigma^{\frac{2}{3}} ; b^{*} \sigma^{\frac{2}{3}}\right)=-\liminf _{n \rightarrow \infty} \frac{1}{n} \log P\left(\left(S_{j}\right)_{j=0}^{n} \text { is SAW, }\left|S_{n}\right| \geq b \sigma^{\frac{2}{3}} n\right) .
$$


Instead of (4.11), now consider

$$
Z_{n}^{\infty}(\mu)=E\left(e^{\mu \sigma^{-\frac{4}{3}} S_{n}} \mathbb{1}_{\left\{\left(S_{j}\right)_{j=0}^{n} \text { is SAW }\right\}}\right), \quad n \in \mathbb{N}, \mu \in \mathbb{R} .
$$

Cut the path into $n / T_{\sigma}$ pieces of length $T_{\sigma}=\sigma^{\frac{2}{3}} T$. (To simplify the notation, assume that both $n / T_{\sigma}$ and $T_{\sigma}$ are integers.) For $\mu>0$, we estimate, like in (4.12)-(4.13),

$$
P\left(\left(S_{j}\right)_{j=0}^{n} \text { is SAW, } S_{n} \geq b \sigma^{\frac{2}{3}} n\right) \leq e^{-\mu b \sigma^{-\frac{2}{3}} n} Z_{n}^{\infty}(\mu) \leq e^{-\mu b \sigma^{-\frac{2}{3}} n}\left[Z_{T_{\sigma}}^{\infty}(\mu)\right]^{n / T_{\sigma}} .
$$

2. The following lemma is the analogue of Lemma 4.2 needed here.

Lemma 5.2. Assume (2.23)-2.24). Then, for any $\mu \in \mathbb{R}$,

$$
\lim _{\sigma \rightarrow \infty} Z_{T_{\sigma}}^{\infty}(\mu)=\widehat{E}\left(e^{-\widehat{H}_{T}} e^{\mu B_{T}}\right) .
$$

Proof of Lemma 5.2. As in the proof of Lemma 4.2, it suffices to show that

$$
\limsup _{\sigma \rightarrow \infty} E\left(e^{2 \mu \sigma^{-\frac{4}{3}} S_{T_{\sigma}}}\right)<\infty \text {. }
$$

Denote the moment generating function of $S_{1} / \sigma$ by $\varphi_{\sigma}(t)=E\left(e^{t S_{1} / \sigma}\right)$. Then

$$
E\left(e^{2 \mu \sigma^{-\frac{4}{3}} S_{T_{\sigma}}}\right)=\varphi_{\sigma}\left(2 \mu \sigma^{-\frac{1}{3}}\right)^{T_{\sigma}} .
$$

By (2.24), the right-hand side is finite for $\sigma$ large enough. By (2.23) (a) we have, uniformly in $\sigma \geq 1$,

$$
\varphi_{\sigma}(t)=1+\frac{1}{2} t^{2}+\mathcal{O}\left(|t|^{3}\right), \quad t \rightarrow 0 .
$$

Put $t=2 \mu \sigma^{-\frac{1}{3}}$ and combine (5.9)-(5.10), to get

$$
E\left(e^{2 \mu \sigma^{-\frac{4}{3}} S_{T_{\sigma}}}\right) \leq e^{T_{\sigma}\left[\frac{1}{2} t^{2}+\mathcal{O}(1 / \sigma)\right]}=e^{2 T \mu^{2}\left[1+\mathcal{O}\left(\sigma^{-\frac{1}{3}}\right)\right]}, \quad \sigma \rightarrow \infty .
$$

3. The details of the remainder of the proof are the same as in Section 4.3.1, via Lemma 5.2 instead of Lemma 4.2. This completes the proof for $b \geq b^{*}$. The proof for $0 \leq b \leq b^{*}$ is analogous.

\subsubsection{Proof of (5.2).}

1. Fix $b \geq b^{*}$. Pick any $b^{\prime}>b$, fix $\sigma, T>0$, and put $\gamma^{(n)}=\gamma_{T} \sigma^{\frac{2}{3}} n / T$. Then, for $\mu>0$ and $T$ large enough, we have

$$
\mathbb{1}_{\left\{S_{n} \geq b \sigma^{\frac{2}{3}} n\right\}} \geq \mathbb{1}_{\left\{\left|S_{n}-b^{\prime} \sigma^{\frac{2}{3}} n\right| \leq \gamma^{(n)}\right\}} e^{\mu \sigma^{-\frac{4}{3}\left[S_{n}-b^{\prime} \sigma^{\frac{2}{3}} n-\gamma^{(n)}\right]}} .
$$

This implies the lower bound

$$
\begin{aligned}
\sigma^{\frac{2}{3}} & \liminf _{n \rightarrow \infty} \frac{1}{n} \log P\left(\left(S_{j}\right)_{j=0}^{n} \text { is SAW, } S_{n} \geq b \sigma^{\frac{2}{3}} n\right) \\
& \geq-\mu b^{\prime}-\mu \frac{\gamma_{T}}{T}+\sigma^{\frac{2}{3}} \liminf _{n \rightarrow \infty} \frac{1}{n} \log E\left(\mathbb{1}_{\left\{\left(S_{j}\right)_{j=0}^{n} \text { is SAW }\right\}} e^{\mu \sigma^{-\frac{4}{3}} S_{n}} \mathbb{1}_{\left\{\left|S_{n}-b^{\prime} \sigma^{\frac{2}{3}} n\right| \leq \gamma^{(n)}\right\}}\right) .
\end{aligned}
$$

To handle the expectation in the right-hand side, we estimate

$$
e^{\mu \sigma^{-\frac{4}{3}} S_{n}} \mathbb{1}_{\left\{\left|S_{n}-b^{\prime} \sigma^{\frac{2}{3}} n\right| \leq \gamma^{(n)}\right\}} \geq \prod_{i=1}^{n / T_{\sigma}}\left[e^{\mu \sigma^{-\frac{4}{3}} S_{T_{\sigma}}^{(i)}} \mathbb{1}_{\left\{\left|\sigma^{-\frac{4}{3}} S_{T_{\sigma}}^{(i)}-b^{\prime} T\right| \leq \gamma_{T}\right\}} \mathbb{1}_{\mathcal{E}_{i}(\delta, T, \sigma)}\right],
$$


where we use the definition (4.27) of the shifted $i$-th piece with $T_{\beta}$ replaced by $T_{\sigma}$, abbreviate $S^{(i)}=$ $\left(S_{j}^{(i)}\right)_{j=0}^{T_{\sigma}}$, and introduce the event

$$
\mathcal{E}_{i}(\delta, T, \sigma)=\left\{S^{(i)} \subset\left[-\delta \sigma^{\frac{2}{3}}, S_{T_{\sigma}}^{(i)}+\delta \sigma^{\frac{2}{3}}\right]\right\} .
$$

2. Assume that $\delta<b T / 2$. On the event $\bigcap_{i=1}^{n / T_{\sigma}} \mathcal{E}_{i}(\delta, T, \sigma)$, the pieces $S^{(i)}, i=1, \ldots, n / T_{\sigma}$, have no mutual intersection, unless they are neighbors of each other. Hence, we only need to estimate the interaction between the neighboring pieces. More precisely, $\left(S_{j}\right)_{j=0}^{n}$ is SAW as soon as all the pieces $S^{(i)}$ are SAW and neighboring pieces do not overlap in more than their connecting point. Introduce the indicator $U_{i}$ of the event that the $i$-th and the $(i+1)$-st piece intersect each other in more than their connecting point:

$$
U_{i}(T, \sigma)= \begin{cases}1 & \text { if }\left(S_{j}\right)_{j=(i-1) T_{\sigma}}^{i T_{\sigma}} \cap\left(S_{j}\right)_{j=i T_{\sigma}}^{(i+1) T_{\sigma}} \neq\left\{S_{i T_{\sigma}}\right\} \\ 0 & \text { otherwise }\end{cases}
$$

Then we have

$$
\mathbb{1}_{\left\{\left(S_{j}\right)_{j=0}^{n} \text { is SAW }\right\}} \prod_{i=1}^{n / T_{\sigma}} \mathbb{1}_{\mathcal{E}_{i}(\delta, T, \sigma)}=\prod_{i=1}^{n / T_{\sigma}}\left[\mathbb{1}_{\left\{S^{(i)} \text { is SAW }\right\}} \mathbb{1}_{\mathcal{E}_{i}(\delta, T, \sigma)}\right] \prod_{i=1}^{n / T_{\sigma}-1}\left(1-U_{i}(T, \sigma)\right) .
$$

Using (5.14) and (5.17), we obtain the lower bound

$$
E\left(\mathbb{1}_{\left\{\left(S_{j}\right)_{j=0}^{n} \text { is SAW }\right\}} e^{\mu \sigma^{-\frac{4}{3}} S_{n}} \mathbb{1}_{\left\{\left|S_{n}-b^{\prime} \sigma^{\frac{2}{3}} n\right| \leq \gamma^{(n)}\right\}}\right) \geq c_{n / T_{\sigma}}\left(\delta, T, \sigma, b^{\prime}, \mu\right),
$$

where

$$
c_{N}=c_{N}\left(\delta, T, \sigma, b^{\prime}, \mu\right)=E\left(\prod_{i=1}^{N-1}\left(1-U_{i}(T, \sigma)\right) \prod_{i=1}^{N} X_{i}\right), \quad N \in \mathbb{N}
$$

with

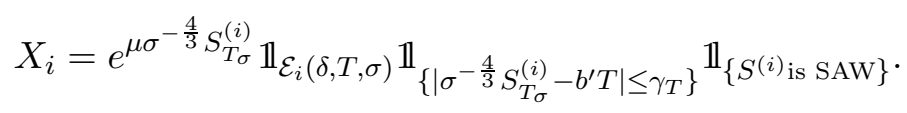

3. Next use an expansion argument that is reminiscent of the "lace expansion technique", namely, expand the product $\prod_{i=1}^{N-1}\left(1-U_{i}\right)$ in 5.19$)$ as

$$
\prod_{i=1}^{N-1}\left(1-U_{i}\right)=\sum_{m=1}^{N} \prod_{i=1}^{m-1}\left(-U_{i}\right) \prod_{i=m+1}^{N-1}\left(1-U_{i}\right)
$$

where the empty product is defined to be equal to 1 . This expansion has the advantage that every summand splits into a product of two separated products. Substitute (5.21) into (5.19), to find that

$$
c_{N}=\sum_{m=1}^{N}(-1)^{m-1} E\left(\left[\prod_{i=1}^{m-1} U_{i} X_{i}\right] \times X_{m} \times\left[\prod_{i=m+1}^{N-1}\left(1-U_{i}\right) X_{i}\right] \times X_{N}\right) .
$$

Since in the $m$-th summand the term $U_{m}$ is absent, the two factors between the two pairs of large square brackets are independent: they depend on the path $\left(S_{j}\right)_{j=0}^{n}$ up time $m T_{\sigma}$, respectively, from time $m T_{\sigma}$ onwards. Hence, the $c_{N}$ satisfy the following renewal relation:

$$
c_{N}=c_{1} c_{N-1}+\sum_{m=2}^{N}(-1)^{m-1} \pi_{m} c_{N-m}, \quad N \in \mathbb{N},
$$


where

$$
\pi_{m}=\pi_{m}\left(\delta, T, \sigma, b^{\prime}, \mu\right)=E\left(\prod_{i=1}^{m-1} U_{i} \prod_{i=1}^{m} X_{i}\right)
$$

4. Use the Cauchy-Schwarz inequality, to estimate

$$
\pi_{m} \leq E\left(\prod_{\substack{i=1 \\ i \text { odd }}}^{m-1} U_{i} \prod_{i=1}^{m} X_{i}\right)^{1 / 2} E\left(\prod_{\substack{i=1 \\ i \text { even }}}^{m-1} U_{i} \prod_{i=1}^{m} X_{i}\right)^{1 / 2}=\left(\pi_{2}^{m / 2}\right)^{1 / 2} c_{1}\left(\pi_{2}^{(m-2) / 2}\right)^{1 / 2}, \quad m \in \mathbb{N} \text { even, }
$$

and similarly for $m \in \mathbb{N}$ odd. Hence

$$
\pi_{m} \leq \varepsilon^{m-1} c_{1}^{m}, \quad m \in \mathbb{N},
$$

where

$$
\varepsilon=\frac{\sqrt{\pi_{2}}}{c_{1}}
$$

5. The following two lemmas give us control over $\varepsilon$ and $c_{N}$. From now on, we choose $\mu=\mu_{b^{\prime}}$ with $\mu_{b^{\prime}}$ the maximizer in (3.6), i.e., $\widehat{I}\left(b^{\prime}\right)=\mu_{b^{\prime}} b^{\prime}-\Lambda^{+}\left(\mu_{b^{\prime}}\right)$, which is possible when $b^{\prime}>b^{* *}$ (recall (3.6)).

Lemma 5.3. Fix $b^{\prime}>b^{* *}$. Then

$$
\lim _{\delta \downarrow 0} \limsup _{T \rightarrow \infty} \limsup _{\sigma \rightarrow \infty} \varepsilon\left(\delta, T, \sigma, b^{\prime}, \mu_{b^{\prime}}\right)=0 .
$$

Lemma 5.4. For $\eta>0$ sufficiently small the following is true: If $\delta, T, \sigma>0$ are chosen such that $\varepsilon=\varepsilon\left(\delta, T, \sigma, b^{\prime}, \mu_{b^{\prime}}\right)<\eta$, then there are numbers $C, N_{0}>0$ (depending on $\varepsilon$ and $\eta$ only) such that

$$
c_{N} \geq C(1-3 \eta)^{N} c_{1}^{N}, \quad N>N_{0} .
$$

6. Before giving the proof of Lemmas 5.3 5.4, we complete the argument. Pick $\eta \in\left(0, \frac{1}{4}\right)$ so small that Lemma 5.4 is satisfied for this $\eta$. According to Lemma 5.3, we may pick $\delta>0$ so small that, when $T$ is picked sufficiently large, we have $\varepsilon<\eta$ for any sufficiently large $\sigma$. Hence we may make use of the estimate in (5.29) for these $T$ and $\sigma$.

We use (5.18) and Lemma 5.4 in (5.13), to obtain

$$
\begin{aligned}
\sigma^{\frac{2}{3}} I_{\infty}^{+}\left(b \sigma^{\frac{2}{3}} ; b^{*} \sigma^{\frac{2}{3}}\right) & =-\sigma^{\frac{2}{3}} \liminf _{n \rightarrow \infty} \frac{1}{n} \log P\left(\left(S_{j}\right)_{j=0}^{n} \text { is SAW, } S_{n} \geq b \sigma^{\frac{2}{3}} n\right) \\
& \leq \mu_{b^{\prime}} b^{\prime}+\mu_{b^{\prime}} \frac{\gamma_{T}}{T}-\liminf _{n \rightarrow \infty} \frac{\sigma^{\frac{2}{3}}}{n} \log c_{n / T_{\sigma}} \\
& \leq \mu_{b^{\prime}} b^{\prime}+\mu_{b^{\prime}} \frac{\gamma_{T}}{T}-\liminf _{n \rightarrow \infty} \frac{\sigma^{\frac{2}{3}}}{n} \log \left[C(1-3 \eta)^{n / T_{\sigma}} c_{1}^{n / T_{\sigma}}\right] \\
& =\mu_{b^{\prime}} b^{\prime}+\mu_{b^{\prime}} \frac{\gamma_{T}}{T}-\frac{1}{T} \log (1-3 \eta)-\frac{1}{T} \log c_{1} .
\end{aligned}
$$

Return to (2.21) and recall that $\left\{H_{T_{\sigma}}=0\right\}=\left\{S^{(1)}\right.$ is SAW $\}$. From the weak convergence assertion in (2.21) applied to (5.19) for $N=1$, in combination with a statement like in Lemma 5.2, it follows that

$$
\lim _{\sigma \rightarrow \infty} c_{1}\left(\delta, T, \sigma, b^{\prime}, \mu_{b^{\prime}}\right)=\widehat{E}\left(e^{-\widehat{H}_{T}} e^{\mu_{b^{\prime}} B_{T}} \mathbb{1}_{\widehat{\mathcal{E}}(\delta, T)} \mathbb{1}_{\left\{B_{T} \approx b^{\prime} T\right\}}\right),
$$


where $\widehat{\mathcal{E}}(\delta, T)$ is the event defined in $(\widehat{3.8})$. Combining (5.30)-(5.31), we obtain

$$
\limsup _{\sigma \rightarrow \infty}\left[\sigma^{\frac{2}{3}} I_{\infty}^{+}\left(b \sigma^{\frac{2}{3}} ; b^{*} \sigma^{\frac{2}{3}}\right)\right] \leq \mu_{b^{\prime}} b^{\prime}+\mu_{b^{\prime}} \frac{\gamma_{T}}{T}-\frac{1}{T} \log (1-3 \eta)-\frac{1}{T} \log \widehat{E}\left(e^{-\widehat{H}_{T}} e^{\mu_{b^{\prime}} B_{T}} \mathbb{1}_{\widehat{\mathcal{E}}(\delta, T)} \mathbb{1}_{\left\{B_{T} \approx b^{\prime} T\right\}}\right) .
$$

Now let $T \rightarrow \infty$ and use (3.11) for $C=\infty$, to see that the right-hand side of (5.32) tends to $\mu_{b^{\prime}} b^{\prime}-\Lambda^{+}\left(\mu_{b^{\prime}}\right)$, which is equal to $\widehat{I}_{1}\left(b^{\prime}\right)$. Finally, let $b^{\prime} \downarrow b$ and use the continuity of $\widehat{I}_{1}$ to finish the proof of (5.2).

\subsection{Proof of Lemma 5.3.}

1. Fix $\delta, T$. Introduce the Brownian event

$$
\widehat{\mathcal{E}}_{i}(\delta, T)=\left\{B_{[(i-1) T, i T]} \subset\left[-\delta+B_{(i-1) T}, B_{i T}+\delta\right]\right\}, \quad i=1,2,
$$

and note that $\widehat{\mathcal{E}}_{i}(\delta, T)$ is identical to $\widehat{\mathcal{E}}(\delta, T)$ in (3.8) for the $i$-th piece. Write $U_{1}$ as $1-\left(1-U_{1}\right)$ in the definition of $\pi_{2}$ in (5.24), to obtain from (5.27) that

$$
\begin{aligned}
\varepsilon^{2} & =\frac{1}{c_{1}^{2}}\left[E\left(X_{1} X_{2}\right)-E\left(\mathbb{1}_{\left\{S^{(1)}, S^{(2)} \text { avoid each other }\right\}} X_{1} X_{2}\right)\right] \\
& \left.=1-\frac{1}{c_{1}^{2}} E\left(\mathbb{1}_{\left\{\left(S_{j}\right)_{j=0}^{\left.2 T_{\sigma} \text { is SAW }\right\}}\right.} X_{1} X_{2}\right)\right] .
\end{aligned}
$$

Now apply the weak convergence statement in (2.21) and recall (5.31), to obtain, analogously to (5.31), that

$$
\lim _{\sigma \rightarrow \infty} \varepsilon^{2}=1-\frac{\widehat{E}\left(e^{-\widehat{H}_{2 T}} e^{\mu B_{2 T}} \mathbb{1}_{\widehat{\mathcal{E}}_{1}(\delta, T) \cap \widehat{\mathcal{E}}_{2}(\delta, T)} \mathbb{1}_{\left\{B_{T} \approx b^{\prime} T\right\}} \mathbb{1}_{\left\{B_{2 T}-B_{T} \approx b^{\prime} T\right\}}\right)}{\widehat{E}\left(e^{-\widehat{H}_{T}} e^{\mu B_{T}} \mathbb{1}_{\widehat{\mathcal{E}}_{1}(\delta, T)} \mathbb{1}_{\left\{B_{T} \geq 0\right\}}\right)^{2}}(1+o(1)),
$$

where $o(1)$ refers to $T \rightarrow \infty$.

2. Denote the intersection local time of the $i$-th piece by $\widehat{H}_{T}^{(i)}$. Then (5.35) reads

$$
\lim _{\sigma \rightarrow \infty} \varepsilon^{2}=\frac{\widehat{E}\left(\left[e^{-\widehat{H}_{T}^{(1)}-\widehat{H}_{T}^{(2)}}-e^{-\widehat{H}_{2 T}}\right] e^{\mu B_{2 T}} \mathbb{1}_{\widehat{\mathcal{E}}_{1}(\delta, T) \cap \widehat{\mathcal{E}}_{2}(\delta, T)} \mathbb{1}_{\left\{B_{T} \approx b^{\prime} T\right\}} \mathbb{1}_{\left\{B_{2 T}-B_{T} \approx b^{\prime} T\right\}}\right.}{\widehat{E}\left(e^{-\widehat{H}_{T}} e^{\mu B_{T}} \mathbb{1}_{\widehat{\mathcal{E}}_{1}(\delta, T)} \mathbb{1}_{\left\{B_{T} \geq 0\right\}}\right)^{2}}(1+o(1)) .
$$

Denote the local time of the $i$-th piece by $L^{(i)}(T, \cdot)$. Then, on the event $\widehat{\mathcal{E}}_{1}(\delta, T) \cap \widehat{\mathcal{E}}_{2}(\delta, T)$, we have

$$
\widehat{H}_{2 T}=\widehat{H}_{T}^{(1)}+\widehat{H}_{T}^{(2)}+2 \int_{B_{T}-\delta}^{B_{T}+\delta} L^{(1)}(T, x) L^{(2)}(T, x) \mathrm{d} x .
$$

Now fix a small $\alpha>0$ and introduce the events

$$
\begin{aligned}
& \widehat{\mathcal{E}}_{1}^{\geq,+}(\delta, \alpha, T)=\left\{\max _{x \in\left[B_{T}-\delta, B_{T}+\delta\right]} L^{(1)}(T, x) \geq \alpha \delta^{-1 / 2}\right\}, \\
& \widehat{\mathcal{E}}_{2}^{\geq,-}(\delta, \alpha, T)=\left\{\max _{x \in\left[B_{T}-\delta, B_{T}+\delta\right]} L^{(2)}(T, x) \geq \alpha \delta^{-1 / 2}\right\} .
\end{aligned}
$$

We estimate the right-hand side of (5.35) differently on the event $\widehat{\mathcal{E}}_{1}^{\geq,+} \cup \widehat{\mathcal{E}}_{2}{ }^{\geq,-}$and on its complement. Namely, on the complement of $\widehat{\mathcal{E}}_{1}^{\geq,+} \cup \widehat{\mathcal{E}}_{2}^{\geq,-}$we estimate

$$
\widehat{H}_{2 T} \leq \widehat{H}_{T}^{(1)}+\widehat{H}_{T}^{(2)}+4 \alpha^{2}
$$

which implies

$$
e^{-\widehat{H}_{T}^{(1)}-\widehat{H}_{T}^{(2)}}-e^{-\widehat{H}_{2 T}} \leq\left[1-e^{-4 \alpha^{2}}\right] e^{-\widehat{H}_{T}^{(1)}-\widehat{H}_{T}^{(2)}},
$$


while on the event $\widehat{\mathcal{E}}_{1}^{\geq,+} \cup \widehat{\mathcal{E}}_{2}^{\geq,-}$we estimate $-e^{-\widehat{H}_{2 T}} \leq 0$. By symmetry, $\widehat{\mathcal{E}}_{1}^{\geq,+}$and $\widehat{\mathcal{E}}_{2}^{\geq,-}$have the same probability. Summarizing, we obtain

$$
\lim _{\sigma \rightarrow \infty} \varepsilon^{2} \leq 1-e^{-4 \alpha^{2}}+2\left(\frac{\widehat{E}\left(e^{-\widehat{H}_{T}} e^{\mu B_{T}} \mathbb{1}_{\widehat{\mathcal{E}}(\delta, T)} \mathbb{1}_{\widehat{\mathcal{E}} \geq(\delta, \alpha, T)} \mathbb{1}_{\left\{B_{T} \approx b^{\prime} T\right\}}\right)}{\widehat{E}\left(e^{-\widehat{H}_{T}} e^{\mu B_{T}} \mathbb{1}_{\widehat{\mathcal{E}}(\delta, T)} \mathbb{1}_{\left\{B_{T} \geq 0\right\}}\right)}\right)^{2}(1+o(1)),
$$

where we recall that the events $\widehat{\mathcal{E}}(\delta, T)$ and $\widehat{\mathcal{E}}^{\geq}(\delta, \alpha, T)$ are defined in (3.8), respectively, (3.10).

3. Let $T \rightarrow \infty$ in 5.42 and use Proposition 3.4(i-ii), to obtain

$$
\limsup _{T \rightarrow \infty} \lim _{\sigma \rightarrow \infty} \varepsilon^{2} \leq 1-e^{-4 \alpha^{2}}+2 \frac{K_{2}(\delta, \alpha)}{K_{1}(\delta, \infty)} .
$$

Let $\delta \downarrow 0$ and use Proposition 3.4(iii), to obtain

$$
\limsup _{\delta \downarrow 0} \limsup _{T \rightarrow \infty} \lim _{\sigma \rightarrow \infty} \varepsilon^{2} \leq 1-e^{-4 \alpha^{2}}
$$

Let $\alpha \downarrow 0$, to arrive at the assertion in (5.28).

\subsection{Proof of Lemma 5.4.}

1. Fix $\eta>0$ and $\varepsilon \in(0, \eta)$. Define $z \in(0, \infty)$ by

$$
1-z=\sum_{m=2}^{\infty}(-1)^{m-1} \pi_{m}\left(\frac{z}{c_{1}}\right)^{m} .
$$

Equation (5.26) implies that, for any $z \in\left(0, \frac{1}{\eta}\right)$, the modulus of the right-hand side is bounded above by $\varepsilon z^{2} /(1-\varepsilon z) \leq \eta z^{2} /(1-\eta z)$. Since this function crosses $1-z$ in $z=\frac{1}{1+\eta}$ and since, for sufficiently small $\eta$, its negative value crosses $1-z$ in $\frac{3}{2}+\mathcal{O}(\eta)$, there is indeed a solution $z$ to (5.45) in $\left(0, \frac{3}{2}+\mathcal{O}(\eta)\right.$ ] as $\eta \downarrow 0$. Assume that $\eta$ is so small that this solution exists and satisfies the estimate $z^{-1} \geq 1-3 \eta$.

2. Abbreviate

$$
A_{N}=c_{N}\left(\frac{z}{c_{1}}\right)^{N}, \quad N \in \mathbb{N} \quad\left(A_{0}=1\right) .
$$

We claim that, if $\eta$ is small enough, then there are numbers $K>0$ and $q \in(0,1)$ (depending on $\eta$ only) such that

$$
\left|A_{N}-A_{N-1}\right| \leq K q^{N}, \quad N \in \mathbb{N} .
$$

The proof of this claim is given in part 3. Because of (5.47), $A_{\infty}=\lim _{N \rightarrow \infty} A_{N} \in(0, \infty)$ exists and, for $N$ sufficiently large, $A_{N} \geq \frac{1}{2} A_{\infty}$, which reads $c_{N} \geq \frac{1}{2} A_{\infty}\left(\frac{c_{1}}{z}\right)^{N}$. Recall that $z^{-1} \geq 1-3 \eta$, to finish the proof of the lemma.

3. The proof of (5.47) goes via induction on $N$. Pick $q=\sqrt{\eta z}$ and assume that $\eta$ is so small that $q<1$ and

$$
\frac{\eta z^{2}}{(1-q)(q-\eta z)} \leq \frac{1}{2}
$$

Furthermore, pick $K \geq 1$ so large that $1+K q /(1-q) \leq K(1-\eta z) / 2 z$. Then the claim holds for $N=1$, since $\left|A_{1}-A_{0}\right|=|z-1| \leq \eta z^{2} /(1-\eta z) \leq \frac{1}{2}(q-\eta z) \leq K q$. Assume now that $N>1$ and that the claim holds for all positive integers $<N$. From this induction hypothesis it follows that, for every $m=2, \ldots, N$,

$$
\left|A_{N-1}-A_{N-m}\right| \leq \sum_{k=2}^{m}\left|A_{N-k+1}-A_{N-k}\right| \leq K q^{N} \sum_{k=2}^{m} q^{-k+1}=\frac{K q}{1-q} q^{N-m} .
$$


Estimate, with the help of $(5.23),(5.26),(5.45)-(5.46)$, the triangle inequality and (5.49),

$$
\begin{aligned}
\left|A_{N}-A_{N-1}\right| & =\left|c_{N-1}\left(\frac{z}{c_{1}}\right)^{N-1}(z-1)+\left(\frac{z}{c_{1}}\right)^{N} \sum_{m=2}^{N}(-1)^{m-1} \pi_{m} c_{N-m}\right| \\
& =\left|-A_{N-1} \sum_{m=2}^{\infty}(-1)^{m-1} \pi_{m}\left(\frac{z}{c_{1}}\right)^{m}+\sum_{m=2}^{N}(-1)^{m-1} \pi_{m}\left(\frac{z}{c_{1}}\right)^{m} A_{N-m}\right| \\
& \leq A_{N-1}\left|\sum_{m=N+1}^{\infty}(-1)^{m-1} \pi_{m}\left(\frac{z}{c_{1}}\right)^{m}\right|+\left|\sum_{m=2}^{N}(-1)^{m-1} \pi_{m}\left(\frac{z}{c_{1}}\right)^{m}\left(A_{N-m}-A_{N-1}\right)\right| \\
& \leq\left(1+\left|A_{N-1}-A_{0}\right|\right) \sum_{m=N+1}^{\infty} \varepsilon^{m-1} z^{m}+\sum_{m=2}^{N} \varepsilon^{m-1} z^{m}\left|A_{N-m}-A_{N-1}\right| \\
& \leq\left(1+\frac{K q}{1-q}\right) \frac{\eta^{N} z^{N+1}}{1-\eta z}+\frac{K q}{1-q} \frac{q^{N}}{\eta} \sum_{m=2}^{\infty}\left(\frac{\eta z}{q}\right)^{m} \\
& =\left(1+\frac{K q}{1-q}\right) \frac{z(\eta z)^{N}}{1-\eta z}+K q^{N} \frac{\eta z^{2}}{(1-q)(q-\eta z)} .
\end{aligned}
$$

Now recall that $1+K q /(1-q) \leq K(1-\eta z) / 2 z$ and recall the estimate in (5.48). Furthermore, observe that $\eta z \leq \sqrt{\eta z}=q$. This implies that the right-hand side of (5.50) is at most $K q^{N}$, which finishes the proof of the induction step.

\section{REMAINING PROOFS}

In this section we prove the remaining results in Section 2: Theorems 2.4 2.6. All the proofs are minor adaptations of the proof in Section 4 .

\subsection{Proof of Theorem 2.4.}

The main result proved in this section is the analogue of Proposition 4.1 for the case where the strength of self-repellence $\beta$ is coupled to the length of the polymer $n$ :

Proposition 6.1. Assume (1.1). If $\beta$ is replaced by $\beta_{n}$ satisfying $\beta_{n} \rightarrow 0$ and $\beta_{n} n^{\frac{3}{2}} \rightarrow \infty$ as $n \rightarrow \infty$, then

$$
\begin{aligned}
-\lim _{n \rightarrow \infty} \frac{1}{\beta_{n}^{\frac{2}{3}} n} \log E\left(e^{-\beta_{n} H_{n}} \mathbb{1}_{\left\{S_{n} \geq b \beta_{n}^{\frac{1}{3}} n\right\}}\right) & =\widehat{I}_{1}^{\sigma}(b), \quad b \geq b^{*} \sigma^{\frac{2}{3}}, \\
-\lim _{n \rightarrow \infty} \frac{1}{\beta_{n}^{\frac{2}{3}} n} \log E\left(e^{-\beta_{n} H_{n}} \mathbb{1}_{\left\{0 \leq S_{n} \leq b \beta_{n}^{\frac{1}{3}} n\right\}}\right) & =\widehat{I}_{1}^{\sigma}(b), \quad b^{* *} \sigma^{\frac{2}{3}}<b \leq b^{*} \sigma^{\frac{2}{3}}, \\
-\liminf _{n \rightarrow \infty} \frac{1}{\beta_{n}^{\frac{2}{3}} n} \log E\left(e^{-\beta_{n} H_{n}} \mathbb{1}_{\left\{0 \leq S_{n} \leq b \beta_{n}^{\frac{1}{3}} n\right\}}\right) & \leq \widehat{I}_{1}^{\sigma}(b), \quad 0 \leq b \leq b^{* *} \sigma^{\frac{2}{3}} .
\end{aligned}
$$

The proof of Proposition 6.1 is identical to that of Proposition 4.1 after we replace the double limit $n \rightarrow \infty, \beta \downarrow 0$ (in this order) by the single limit $n \rightarrow \infty$ with the restrictions $\beta_{n} \rightarrow 0, \beta_{n} n^{\frac{3}{2}} \rightarrow \infty$. The latter implies that $T_{\beta_{n}}=\beta_{n}^{-\frac{2}{3}} T=o(n)$, and it is actually only this fact that is needed in the proof. Therefore we can simply copy the proofs in Sections 4.3.1 4.3.2 to derive Proposition 6.1. The reader is asked to check the details. Proposition 6.1 in turns implies Theorem 2.4(i).

A similar result holds when $\sigma$ is coupled to $n$ with the restrictions $\sigma_{n} \rightarrow \infty, \sigma_{n} n^{-\frac{3}{2}} \rightarrow 0$. The latter implies that $T_{\sigma_{n}}=\sigma_{n}^{\frac{2}{3}} T=o(n)$. The result in turn implies Theorem 2.4(ii). 


\subsection{Proof of Theorem 2.5.}

Define rate functions $I_{\beta, \gamma}^{+}$and $I_{\beta, \gamma}^{-}$as in (4.1) with $\beta H_{n}$ replaced by $H_{n}^{\beta, \gamma}$. Recall (2.9). The main result in this section is the following.

Proposition 6.2. Fix $\sigma \in(0, \infty)$. Then, under (1.1),

$$
\begin{aligned}
& \liminf _{\beta, \gamma}(\beta-\gamma)^{-\frac{2}{3}} I_{\beta, \gamma}^{-}\left(b(\beta-\gamma)^{\frac{1}{3}} ; b^{*}(\beta-\gamma)^{\frac{1}{3}} \sigma^{\frac{2}{3}}\right) \geq \widehat{I}_{1}^{\sigma}(b), \quad b \geq 0, \\
& \limsup _{\beta, \gamma}(\beta-\gamma)^{-\frac{2}{3}} I_{\beta, \gamma}^{+}\left(b(\beta-\gamma)^{\frac{1}{3}} ; b^{*}(\beta-\gamma)^{\frac{1}{3}} \sigma^{\frac{2}{3}}\right) \leq \widehat{I}_{1}^{\sigma}(b), \quad b>b^{* *} \sigma^{\frac{2}{3}} .
\end{aligned}
$$

Proposition 6.2 implies Theorem 2.5, analogously to the proof in Section 4.1. We believe that Proposition 6.2 and Theorem 2.5 fail without the restrictions on $\beta, \gamma$ in (2.9).

\subsubsection{Proof of (6.4).}

Fix $b \geq b^{*} \sigma^{\frac{2}{3}}$. Fix $T>0$ and put $T_{\beta, \gamma}=T_{\beta-\gamma}=T(\beta-\gamma)^{-\frac{2}{3}}$. (Again, assume for notational convenience that both $T_{\beta-\gamma}$ and $n / T_{\beta-\gamma}$ are integers.) First note that the interaction in (2.8) may be written as

$$
H_{n}^{\beta, \gamma}=(\beta-\gamma) H_{n}+\frac{\gamma}{2} G_{n}
$$

where $H_{n}$ is the interaction of the Domb-Joyce model in (1.4), and

$$
G_{n}=\sum_{x \in \mathbb{Z}}\left[\ell_{n}(x)-\ell_{n}(x+1)\right]^{2} .
$$

(Absorb the terms $n+1$ in (1.4) and $\beta(n+1)$ in (2.8) into the normalization.) Define

$$
Y_{n}^{\beta, \gamma}(b)=E\left(e^{-H_{n}^{\beta, \gamma}} \mathbb{1}_{\left\{S_{n} \geq b(\beta-\gamma)^{\frac{1}{3}} n\right\}}\right) .
$$

To get the lower bound, simply estimate $H_{n}^{\beta, \gamma} \geq(\beta-\gamma) H_{n}$ in (6.6), which implies that $Y_{n}^{\beta, \gamma}(b) \leq$ $Y_{n}^{\beta-\gamma, 0}(b)$. Hence

$$
\limsup _{n \rightarrow \infty} \frac{1}{n} \log Y_{n}^{\beta, \gamma}(b) \leq \limsup _{n \rightarrow \infty} \frac{1}{n} \log E\left(e^{-(\beta-\gamma) H_{n}} \mathbb{1}_{\left\{S_{n} \geq b(\beta-\gamma)^{\frac{1}{3}} n\right\}}\right) .
$$

The right-hand side is nothing but the approximative rate function $I_{\beta}^{+}$defined in (4.1) with $\beta$ replaced by $\beta-\gamma$. Hence, (6.4) follows from (4.3).

6.2.2 Proof of (6.5).

1. Like in Section 4.3.2, we first show that (recall 4.35))

$$
\begin{aligned}
& (\beta-\gamma)^{-\frac{2}{3}} I_{\beta, \gamma}^{+}\left(b(\beta-\gamma)^{-\frac{2}{3}} ; b^{*}(\beta-\gamma)^{\frac{1}{3}} \sigma^{\frac{2}{3}}\right) \\
& =-(\beta-\gamma)^{-\frac{2}{3}} \liminf _{n \rightarrow \infty} \frac{1}{n} \log Y_{n}^{\beta, \gamma}(b)
\end{aligned}
$$

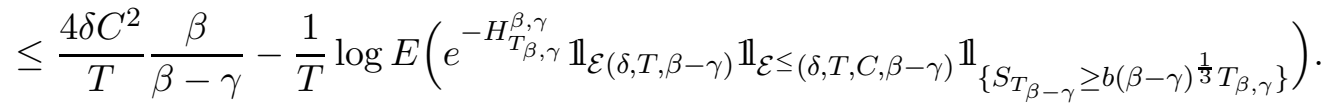

With the help of (6.6) for $n=T_{\beta-\gamma}$ and the inequality $e^{-x} \geq 1-x$, we estimate

$$
e^{-H_{T_{\beta, \gamma}, \gamma}^{\beta, \gamma}}=e^{-(\beta-\gamma) H_{T_{\beta-\gamma}}} e^{-\frac{\gamma}{2} G_{T_{\beta-\gamma}}} \geq e^{-(\beta-\gamma) H_{T_{\beta-\gamma}}}\left[1-\frac{\gamma}{2} G_{T_{\beta-\gamma}}\right] \geq e^{-(\beta-\gamma) H_{T_{\beta-\gamma}}}-\frac{\gamma}{2} G_{T_{\beta-\gamma}} .
$$

As to the second term on the right-hand side of (6.11), in part 2 we show that

$$
\lim _{\beta, \gamma} \frac{\gamma}{2} E\left(G_{T_{\beta-\gamma}}\right)=0 \text {, }
$$


where $\lim _{\beta, \gamma}$ is the limit in (2.9). Hence, applying $\lim _{\beta, \gamma}$ on the right-hand side of (6.10), we see that the remainder of the proof is now the same as in Section 4.3.1 after (4.35). Thus, the proof is finished as soon as (6.12) is proved.

2. In order to prove 6.12 , we compute

$$
\begin{aligned}
E\left(\sum_{x \in \mathbb{Z}}\left[\ell_{n}(x)-\ell_{n}(x+1)\right]^{2}\right) & =\sum_{i, j=0}^{n}\left[2 P\left(S_{i}=S_{j}\right)-P\left(S_{i}+1=S_{j}\right)-P\left(S_{i}-1=S_{j}\right)\right] \\
& =\sum_{i, j=0}^{n}\left[2 P\left(S_{|i-j|}=0\right)-P\left(S_{|i-j|}=1\right)-P\left(S_{|i-j|}=-1\right)\right] \\
& =2 n+\sum_{k=1}^{n} \sum_{j=0}^{n-k}\left[2 P\left(S_{k}=0\right)-P\left(S_{k}=1\right)-P\left(S_{k}=-1\right)\right] \\
& =2 n+\sum_{k=1}^{n}(n-k+1)\left[2 P\left(S_{k}=0\right)-P\left(S_{k}=1\right)-P\left(S_{k}=-1\right)\right] .
\end{aligned}
$$

We must show that the right-hand side of $(\sqrt{6.13})$ is $\mathcal{O}(n)$, because then $(6.12)$ follows via our assumption that $\gamma(\beta-\gamma)^{-\frac{2}{3}} \rightarrow 0$. This is shown in Lemma 6.3 below.

Lemma 6.3. As $n \rightarrow \infty$,

$$
\sum_{k=1}^{n}(n-k+1)\left[2 P\left(S_{k}=0\right)-P\left(S_{k}=1\right)-P\left(S_{k}=-1\right)\right]=\mathcal{O}(n) .
$$

Proof of Lemma 6.3. Let $\phi(t)=E\left(e^{\mathrm{i} t S_{1}}\right)$ denote the characteristic function of $S_{1}$. We have

$$
P\left(S_{k}=x\right)=\frac{1}{2 \pi} \int_{-\pi}^{\pi} e^{\mathrm{i} t x} \phi(t)^{k} \mathrm{~d} t, \quad x \in \mathbb{Z}, k \in \mathbb{N} .
$$

In particular,

$$
2 P\left(S_{k}=0\right)-P\left(S_{k}=1\right)-P\left(S_{k}=-1\right)=\frac{1}{\pi} \int_{-\pi}^{\pi}[1-\cos t] \phi(t)^{k} \mathrm{~d} t .
$$

Abbreviate the left-hand side of (6.14) by $B_{n}$. Then (6.16) says that

$$
B_{n}=\frac{1}{\pi} \int_{-\pi}^{\pi}\left[[1-\cos t] \sum_{k=1}^{n}(n+1-k) \phi(t)^{k}\right] \mathrm{d} t .
$$

We next use that

$$
\sum_{k=1}^{n}(n+1-k) \phi^{k}=\frac{n \phi}{1-\phi}-\phi \frac{1-\phi^{n}}{[1-\phi]^{2}}, \quad \text { on }\{\phi \neq 1\}
$$

to arrive at

$$
B_{n}=n \frac{1}{\pi} \int_{-\pi}^{\pi} \phi(t) \frac{1-\cos t}{1-\phi(t)} \mathrm{d} t-\frac{1}{\pi} \int_{-\pi}^{\pi}\left[\phi(t) \frac{1-\cos t}{1-\phi(t)} \frac{1-\phi^{n}(t)}{1-\phi(t)}\right] \mathrm{d} t .
$$

For the first term, we use that $|\phi(t)| \leq 1, t \in[-\pi, \pi]$, and that the map $t \mapsto \frac{1-\cos t}{1-\phi(t)}$ is bounded on $[-\pi, \pi] \backslash\{0\}$, since the only value where $\phi(t)=1$ is $t=0$. This shows that the first term is of order $\mathcal{O}(n)$. For the second term, we use that

$$
\left|\frac{1-\phi^{n}(t)}{1-\phi(t)}\right|=\left|\sum_{k=0}^{n-1} \phi^{k}(t)\right| \leq n, \quad t \in[-\pi, \pi] \backslash\{0\},
$$


so that also the second term in $(6.19)$ is of order $\mathcal{O}(n)$.

\subsection{Proof of Theorem 2.6.}

Let $I_{L}^{+}$and $I_{L}^{-}$denote the two approximative rate functions for the endpoint of the first coordinate, $S_{n}$, with the convention $e^{-\infty H_{n}}=\mathbb{1}_{\left\{H_{n}=0\right\}}$, i.e.,

$$
I_{L}^{+}(\theta ; \widetilde{\theta})= \begin{cases}-\liminf _{n \rightarrow \infty} \frac{1}{n} \log P^{L}\left(H_{n}=0, S_{n} \geq \theta n\right) & \text { if } \theta \geq \widetilde{\theta} \\ -\liminf _{n \rightarrow \infty} \frac{1}{n} \log P^{L}\left(H_{n}=0,0 \leq S_{n} \leq \theta n\right) & \text { if } 0 \leq \theta \leq \widetilde{\theta}\end{cases}
$$

and similarly for $I_{L}^{-}$with limsup. The result below identifies the asymptotics of these rate functions in the limit as $n \rightarrow \infty$ followed by $L \rightarrow \infty$, and also when the two limits are coupled in a certain way:

Proposition 6.4. Fix $\sigma \in(0, \infty)$ and assume (1.1).

(i) Then

$$
\begin{aligned}
\liminf _{L \rightarrow \infty} L^{\frac{2}{3}} I_{L}^{-}\left(b(4 L)^{-\frac{1}{3}} ; b^{*}(4 L)^{-\frac{1}{3}} \sigma^{\frac{2}{3}}\right) & \geq \widehat{I}_{1}^{\sigma}(b), \quad b \geq 0, \\
\limsup _{L \rightarrow \infty} L^{\frac{2}{3}} I_{L}^{+}\left(b(4 L)^{-\frac{1}{3}} ; b^{*}(4 L)^{-\frac{1}{3}} \sigma^{\frac{2}{3}}\right) & \leq \widehat{I}_{1}^{\sigma}(b), \quad b>b^{* *} \sigma^{\frac{2}{3}} .
\end{aligned}
$$

(ii) If $L$ is replaced by $L_{n}$ satisfying $L_{n} \rightarrow \infty$ and $L_{n} n^{-\frac{3}{2}} \rightarrow 0$ as $n \rightarrow \infty$, then

$$
\begin{gathered}
-\lim _{n \rightarrow \infty} \frac{1}{\left(4 L_{n}\right)^{-\frac{2}{3}} n} \log P^{L_{n}}\left(H_{n}=0, S_{n} \geq b\left(4 L_{n}\right)^{-\frac{1}{3}} n\right)=\widehat{I}_{1}^{\sigma}(b), \quad b \geq b^{*} \sigma^{\frac{2}{3}} \\
-\lim _{n \rightarrow \infty} \frac{1}{\left(4 L_{n}\right)^{-\frac{2}{3}} n} \log P^{L_{n}}\left(H_{n}=0,0 \leq S_{n} \leq b\left(4 L_{n}\right)^{-\frac{1}{3}} n\right)=\widehat{I}_{1}^{\sigma}(b), \quad b^{* *} \sigma^{\frac{2}{3}}<b \leq b^{*} \sigma^{\frac{2}{3}}(6 \\
-\liminf _{n \rightarrow \infty} \frac{1}{\left(4 L_{n}\right)^{-\frac{2}{3}} n} \log P^{L_{n}}\left(H_{n}=0,0 \leq S_{n} \leq b\left(4 L_{n}\right)^{-\frac{1}{3}} n\right) \leq \widehat{I}_{1}^{\sigma}(b), \quad 0 \leq b \leq b^{* *} \sigma^{\frac{2}{3}}
\end{gathered}
$$

Analogously to before, Theorem 2.6 is implied by Proposition 6.4.

\section{Proof of Proposition 6.4.}

1. Let us compute the conditional probability of the event $\left\{H_{n}=0\right\}$, i.e., the path $\left(X_{0}, \ldots, X_{n}\right)$ is self-avoiding, given the path $S=\left(S_{0}, \ldots, S_{n}\right)$ of the first coordinate. Given $S$, the event $\left\{H_{n}=0\right\}$ is equal to the event that $U_{i}^{L} \neq U_{j}^{L}$ for all time pairs $0 \leq i<j \leq n$ at which $S_{i}=S_{j}$. Let us denote by $\ell_{n}(x), x \in \mathbb{Z}$, the local times of $S$ as in (1.5), and by $i_{1}^{x}, \ldots, i_{\ell_{n}(x)}^{x}$ the times at which $S$ hits $x$. Then $\left\{H_{n}=0\right\}$ is the event that, for all $x \in \mathbb{Z}$, the random variables $U_{i_{1}^{x}}^{L}, \ldots, U_{i_{\ell_{n}(x)}^{x}}^{L}$ are distinct. Since $U_{0}^{L}, \ldots, U_{n}^{L}$ are i.i.d. and uniform on $\{-L, \ldots, L\}$, the conditional probability of this event is easily computed:

$$
P^{L}\left(H_{n}=0 \mid S\right)=\prod_{x \in \mathbb{Z}} \prod_{k=0}^{\ell_{n}(x)-1}\left(1-\frac{k}{2 L+1}\right)=\exp \left\{\sum_{x \in \mathbb{Z}} \sum_{k=0}^{\ell_{n}(x)-1} \log \left(1-\frac{k}{2 L+1}\right)\right\} .
$$


2. Fix $b \geq b^{*} \sigma^{\frac{2}{3}}$. To prove $(6.23)$, use the inequality $\log (1-x) \leq-x$ and the fact that $\sum_{k=0}^{l-1} k=$ $\frac{1}{2} l(l-1)$, to estimate

$$
\begin{aligned}
P^{L}\left(H_{n}=0, S_{n} \geq b(4 L)^{-\frac{1}{3}} n\right) & =E^{L}\left(\mathbb{1}_{\left\{S_{n} \geq b(4 L)^{-\frac{1}{3}} n\right\}} P^{L}\left(H_{n}=0 \mid S\right)\right) \\
& \leq E^{L}\left(\exp \left\{-\frac{1}{4 L+2} \sum_{x \in \mathbb{Z}} \ell_{n}(x)\left[\ell_{n}(x)-1\right]\right\} \mathbb{1}_{\left\{S_{n} \geq b(4 L)^{-\frac{1}{3}} n\right\}}\right) \\
& =E^{L}\left(e^{-\frac{1}{4 L+2} H_{n}} \mathbb{1}_{\left\{S_{n} \geq b(4 L)^{-\frac{1}{3}} n\right\}}\right),
\end{aligned}
$$

with $H_{n}$ denoting the self-intersection local time of $S$ as in (1.4). The right-hand side of (6.28) is nothing but the quantity appearing in (4.1) for the Domb-Joyce model with strength of self-repellence $\beta=\frac{1}{4 L+2}$. For $0 \leq b \leq b^{* *} \sigma^{\frac{2}{3}}$, the same argument works with $\leq$ replacing $\geq$. Hence, (6.22) directly follows from Proposition 4.1.

3. Fix $b>b^{* *} \sigma^{\frac{2}{3}}$. To prove (6.23), we insert the condition that $\max _{x \in \mathbb{Z}} \ell_{n}(x) \leq \sqrt{L}$. We then have that, for all $0 \leq k\left(<\ell_{n}(x)\right) \leq \sqrt{L}$ and $L$ sufficiently large,

$$
\log \left(1-\frac{k}{2 L+1}\right) \geq-\frac{k}{2 L+1}\left(1-\frac{k}{L}\right) \geq-\frac{k}{2 L+1}\left(1-\frac{1}{\sqrt{L}}\right),
$$

and substituting this into 6.27 we get that

$$
P^{L}\left(H_{n}=0, S_{n} \geq b(4 L)^{-\frac{1}{3}} n\right) \geq E^{L}\left(e^{-\frac{1}{4 L+2}\left(1-\frac{1}{\sqrt{L}}\right) H_{n}} \mathbb{1}_{\left\{S_{n} \geq b(4 L)^{-\frac{1}{3}} n\right\}} \mathbb{1}_{\left\{\max _{x} \in \mathbb{Z} \ell_{n}(x) \leq \sqrt{L}\right\}}\right) .
$$

Now we can follow the same argument as in Section 4.3.2, noting that the condition $\max _{x \in \mathbb{Z}} \ell_{n}(x) \leq \sqrt{L}$ is asymptotically negligible as $L \rightarrow \infty$.

4. The proof for $L=L_{n}$ is identical to the above proof and relies on Proposition 6.1.

\section{Discussion.}

The weak interaction limit results in Section 2.1 2.3 were proved in Sections 46 with the help of a new and flexible method. The idea was to cut the path into pieces of an appropriately scaled length, to control the interaction between the different pieces, and to apply the invariance principle to the single pieces. This method allowed us to prove scaling of the large deviation rate function for the empirical drift of the path, which in turn implied the weak interaction limit results in Section 2.1 2.3.

Our method has a number of advantages over the approach that was followed in our earlier work, which relied on a variational representation for the quantities in the central limit theorem and a functional analytic proof that this variational representation scales to a limit. Our new method is simple, works for a very large class of random walks in a variety of self-repelling and self-attracting situations, and allows for a coupled limit in which $n \rightarrow \infty$ and $\beta \downarrow 0$, respectively, $\sigma \rightarrow \infty$ together. We expect that it can be applied to other polymer models as well, such as branched polymers and heteropolymers, which we hope to investigate in the future.

The results in Section 2.1 2.3 show universality, in the sense that the scaling limits do not depend on the details of the underlying random walk other than its step variance and are all given in terms of the Edwards model.

Two items remained open. First, we did not prove the scaling of the rate function in the linear regime (recall the remark at the end of Sections 4.1 and 5.1). In this regime we only derived the upper bound. We have no doubt that the lower bound can be derived too, but this would require some further refinements. In particular, in the linear regime the path makes an overshoot, and we would need to control the interaction between overlapping pieces in the overshoot. Second, we did 
not prove the scaling of the variance in the central limit theorem (recall (2.5)). This would require control of the second derivative of the rate function in its minimum (compare Theorem 3.1(iii) with Theorem 3.2(iii)). We only have good control over the first derivative of the rate function. The LDP does not imply the CLT, so even if we had obtained the scaling of the variance, we would not be able to deduce the CLT anyway.

\section{REFERENCES}

[A86] D. Aldous, Self-intersections of 1-dimensional random walks, Probab. Theory Relat. Fields 72, 559-587, 1986.

[AJ90] S.E. Alm and S. Janson, Random self-avoiding walks on one-dimensional lattices, Commun. Statist.Stochastic Models 6, 169-212, 1990.

[BS95] D.C. BRydges and G. Slade, The diffusive phase of a model of self-interacting walks, Probab. Theory Relat. Fields 103, 285-315, 1995.

[CR83] E. Csáky and P. RÉvesz, Strong invariance for local times, Probab. Theory Relat. Fields 62, 263-278, 1983.

[GH93] A. GReven and F. Den Hollander, A variational characterization of the speed of a one-dimensional selfrepellent random walk, Ann. Appl. Probab. 3, 1067-1099, 1993.

[vdH98] R. VAN DER HOFSTAD, On the constants in the central limit theorem for the one-dimensional Edwards model, J. Stat. Phys. 90, 1295-1306, 1998.

[vdHdH95] R. van der Hofstad and F. Den Hollander, Scaling for a random polymer, Comm. Math. Phys. 169, 397-440, 1995.

[vdHdHK97a] R. van der Hofstad, F. Den Hollander and W. König, Central limit theorem for the Edwards model, Ann. Probab. 25, 573-597, 1997.

[vdHdHK97b] R. van der Hofstad, F. Den Hollander and W. König, Central limit theorem for a weakly interacting random polymer, Markov Proc. Relat. Fields 3, 1-62, 1997.

[vdHdHK02] R. van Der Hofstad, F. Den Hollander and W. König, Large deviations for the Edwards model, preprint 2002.

[vdHK00] R. van Der Hofstad and A. Klenke, Self-attractive random polymers, preprint 2000, to appear in Ann. Appl. Probab.

[vdHK01] R. van Der Hofstad and W. KÖNIG, A survey of one-dimensional random polymers, J. Stat. Phys. 103, 915-944, 2001.

[dH00] F. Den Hollander, Large Deviations, Fields Institute Monographs, American Mathematical Society, Providence (Rhode Island), 2000.

[K93] W. KöNIG, The drift of a one-dimensional self-avoiding random walk, Probab. Theory Relat. Fields 96, 521-543, 1993.

[K94] W. KÖNIG, The drift of a one-dimensional self-repellent random walk with bounded increments, Probab. Theory Relat. Fields 100, 513-544, 1994.

[K96] W. KÖNIG, A central limit theorem for a one-dimensional polymer measure. Ann. Probab. 24, 1012-1035, 1996.

[MS93] N. Madras and G. Slade, The Self-Avoiding Walk, Birkhäuser, Boston, 1993.

[vdZ98] C. Vanderzande, Lattice Models of Polymers, Cambridge University Press, Cambridge, 1998.

[W84] J. Westwater, On Edwards model for polymer chains, in: Trends and Developments in the Eighties (S. Albeverio and P. Blanchard, eds.), Bielefeld Encounters in Math. Phys. 4/5, World Scientific, Singapore, 1984. 